\title{
Inhibition of the GAS6/AXL pathway augments the efficacy of chemotherapies
}

\author{
Mihalis S. Kariolis, ${ }^{1}$ Yu Rebecca Miao, ${ }^{1}$ Anh Diep, ${ }^{1}$ Shannon E. Nash, ${ }^{1}$ Monica M. Olcina, ${ }^{1}$ Dadi Jiang, ${ }^{1}$ Douglas S. Jones II, ${ }^{2}$ \\ Shiven Kapur, ${ }^{2}$ Irimpan I. Mathews, ${ }^{3}$ Albert C. Koong, ${ }^{1}$ Erinn B. Rankin, ${ }^{1}$ Jennifer R. Cochran, ${ }^{2}$ and Amato J. Giaccia ${ }^{1}$ \\ 'Department of Radiation Oncology, Stanford University School of Medicine, and ${ }^{2}$ Department of Bioengineering, Stanford University, Stanford, California, USA. ${ }^{3}$ Stanford Synchrotron Radiation Lightsource, \\ SLAC National Accelerator Laboratory, Menlo Park, California, USA.
}

\begin{abstract}
The AXL receptor and its activating ligand, growth arrest-specific 6 (GAS6), are important drivers of metastasis and therapeutic resistance in human cancers. Given the critical roles that GAS6 and AXL play in refractory disease, this signaling axis represents an attractive target for therapeutic intervention. However, the strong picomolar binding affinity between CAS6 and AXL and the promiscuity of small molecule inhibitors represent important challenges faced by current anti-AXL therapeutics. Here, we have addressed these obstacles by engineering a second-generation, high-affinity $A X L$ decoy receptor with an apparent affinity of 93 femtomolar to GAS6. Our decoy receptor, MYD1-72, profoundly inhibited disease progression in aggressive preclinical models of human cancers and induced cell killing in leukemia cells. When directly compared with the most advanced anti-AXL small molecules in the clinic, MYD1-72 achieved superior antitumor efficacy while displaying no toxicity. Moreover, we uncovered a relationship between AXL and the cellular response to DNA damage whereby abrogation of AXL signaling leads to accumulation of the DNA-damage markers $\gamma \mathrm{H} 2 \mathrm{AX}$, 53BP1, and RAD51. MYD1-72 exploited this relationship, leading to improvements upon the therapeutic index of current standard-of-care chemotherapies in preclinical models of advanced pancreatic and ovarian cancer.
\end{abstract}

\section{Introduction}

With few exceptions, curative treatment protocols in clinical oncology remain reliant upon a combination of surgical resection, ionizing radiation, and cytotoxic chemotherapy. However, in many cases, the full potential of these modalities is limited by off-target effects and dose-limiting toxicities. Even when side effects can be effectively managed, durable responses are difficult to achieve, particularly in cases characterized by refractory, metastatic disease. To address these shortcomings, there has been a trend in drug discovery to develop targeted therapies capable of modulating signaling axes dysregulated in cancers. There are now many FDA-approved Abs (1) and small molecules (2) that allow for therapeutic manipulation of a myriad of clinically relevant targets. Collectively, these drugs have proven beneficial but not transformative (3); metrics of efficacy are often measured in progression-free survival rather than improved overall survival. To date, the complex biology that drives tumorigenesis has been, for the most part, unyielding to single-agent, targeted treatments.

While limited as monotherapies, the value of these drugs lies in their ability to be used with the classic aforementioned treatment modalities. By augmenting standard treatment protocols

Authorship note: M.S. Kariolis and Y.R. Miao contributed equally to this work. Conflict of interest: Stanford University holds a patent "Biologic inhibitors for therapeutic targeting the receptor tyrosine kinase AXL" (US8618254 B2), which is related to the work described in this paper, with M.S. Kariolis, Y.R. Miao, D.S. Jones, E.B. Rankin, J.R. Cochran, and A.J. Giaccia named as inventors. A.J. Giaccia and A.C. Koong are cofounders of Ruga Corp., a company that has licensed this patent.

Submitted: November 16, 2015; Accepted: October 18, 2016.

Reference information: J Clin Invest. 2017;127(1):183-198. doi:10.1172/JCI85610. with inhibitors targeting signaling pathways known to be important within a particular patient, meaningful improvements in efficacy have been obtained within a small subset of individuals. However, most patients remain refractory even to these combination treatments, emphasizing the need for new molecular entities that have direct antitumor activity, but more importantly, act synergistically with surgery, radiation, and/or chemotherapy.

One target that has shown promise in many cancers is AXL, a member of the TAM family of receptor tyrosine kinases that also includes TYRO3 and MER $(4,5)$. Upregulated in many forms of cancer (6), AXL overexpression has been linked to metastasis (7, 8), poor survival (9-11), and drug resistance (12,13). Critically, AXL-deficient mice have mild phenotypes (14), suggesting complete abrogation of signaling through the AXL receptor would confer minimal on-target toxicity. Furthermore, AXL has a single ligand, growth arrest-specific 6 (GAS6) $(15,16)$, and constitutive activation is rarely observed in tumors, leaving GAS6-mediated signaling as the primary driver of pathogenesis. Nevertheless, an unusually strong binding affinity between GAS6 and AXL of approximately $30 \mathrm{pM}(17)$ has made the development of competitive antagonists challenging.

We showed that administration of a soluble AXL decoy receptor (18-21) is an effective therapeutic strategy that circumvents the native affinity barrier (Figure 1A). The AXL receptor contains 2 distinct GAS6-binding epitopes: a high affinity site on its $\mathrm{N}$-terminal Ig-like domain and a low affinity site on the second Ig domain (22). Previously, we engineered the major site on AXL Ig1 using a combination of rational and combinatorial protein-engineering methods (17). The result of these efforts was MYD1, a high-affinity AXL variant containing 4 mutations that conferred improved binding 
A

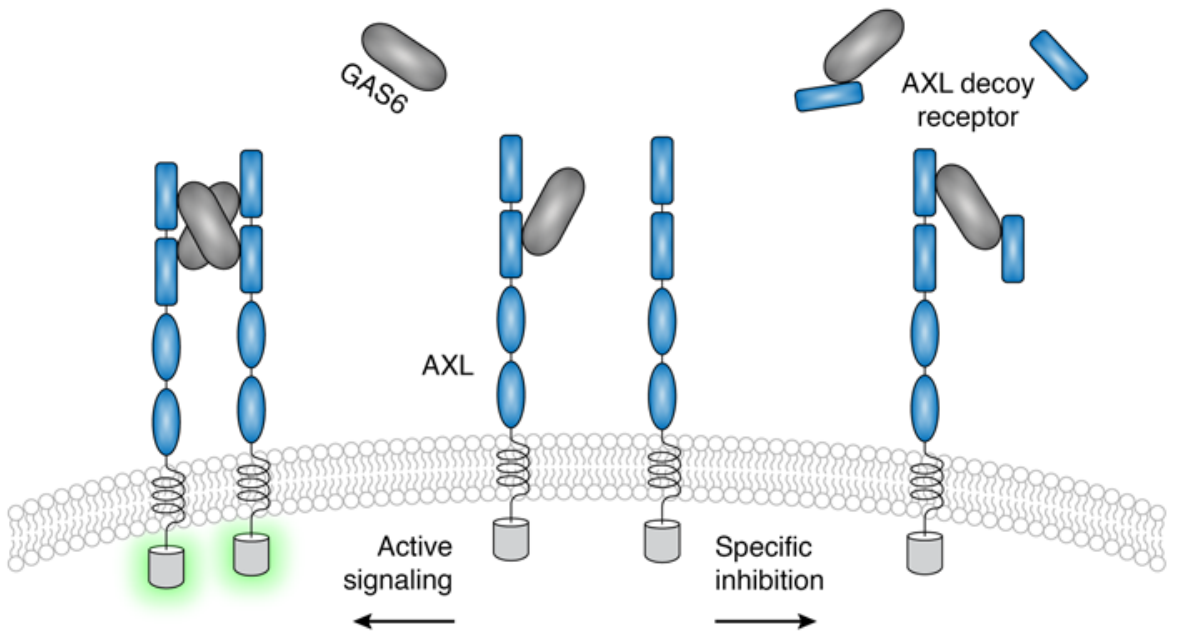

B
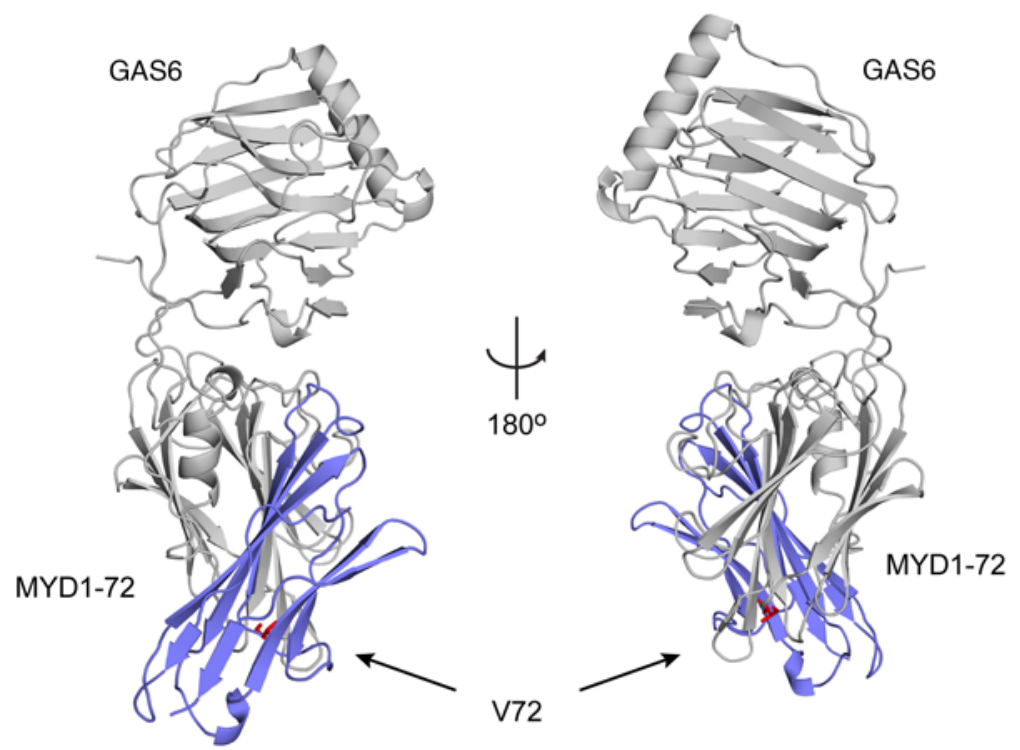

C
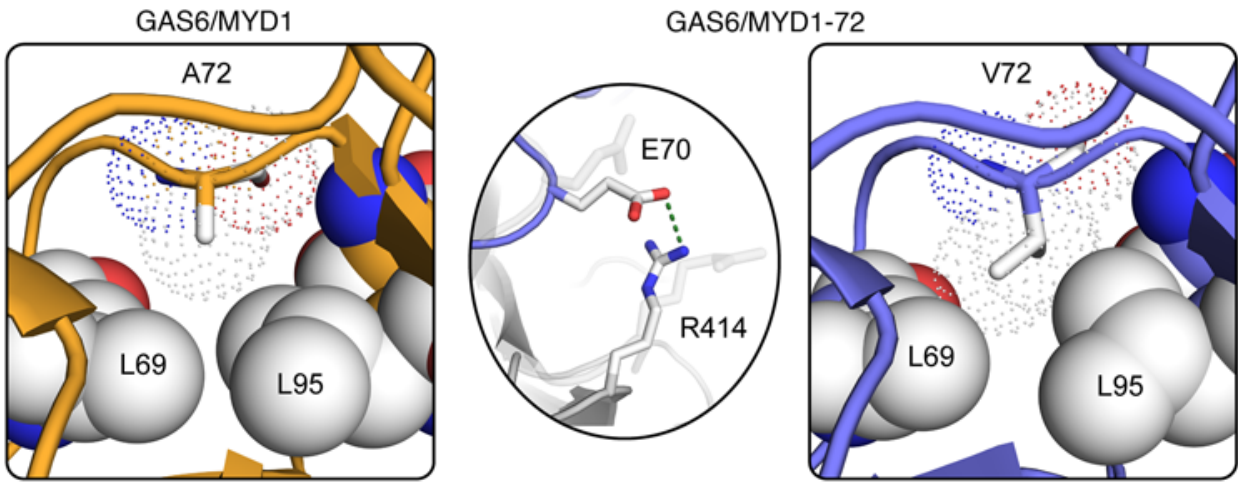

Figure 1. Engineering and characterization of a second-generation AXL decoy receptor. (A) The first immunoglobulin domain of the AXL receptor was engineered for improved affinity to GAS6. When administered, the engineered soluble AXL sequesters GAS6, preventing it from binding to and activating endogenous cell surface-expressed AXL. (B) GAS6/MYD1-72 1:1 cocomplex. GAS6 is shown in gray and MYD1-72 in blue. V72 is highlighted in red, and its location on the structure is indicated (arrows). (c) Cutaway showing A72 on the MYD1 and V72 on the MYD1-72. The sidechains of both are shown as dotted spheres, illustrating the space occupied by the larger valine mutation. The new interaction gained in the MYD1-72 structure is shown in the middle.

both engineered proteins, we further define the correlation between the affinity of the decoy to GAS6 and antitumor efficacy achieved in vivo. The second-generation decoy receptor was well tolerated and outperformed the current lead clinical AXL small molecule inhibitor when directly compared. Finally, we uncovered a relationship between AXL and the DNAdamage response and leveraged this to improve the therapeutic index of standard-of-care cytotoxic chemotherapies in preclinical models of pancreatic and ovarian cancers.

\section{Results}

Engineering a second-generation decoy receptor. In our original work, an error-prone library was created using the WT AXL Ig1 domain as a template and placed into the yeast display system (23). After 6 rounds of flow cytometric sorting, the library was enriched for $3 \mathrm{AXL}$ variants with improved binding to GAS6 (17). While only 3 variants were present after the sixth and final round of sorting, substantial diversity was retained in earlier sort products.

To evaluate whether further increased GAS6-binding affinto GAS6. The characterization of MYD1 revealed a strong correlation between GAS6-binding affinity and therapeutic efficacy of the AXL decoy receptor in preclinical models of cancer metastasis (17).

Here, we report the engineering and characterization of a second-generation AXL decoy receptor that binds both mouse and human GAS6 more tightly than our original molecule. Using ity could improve antitumor efficacy, we performed sequence analysis on the sort 5 products to identify additional gain-offunction mutations. A total of 141 clones were sequenced, yielding 25 unique variants. Three mutations were found to occur in at least $20 \%$ of these unique variants: A72V, D87G, and V92A, the latter 2 
A

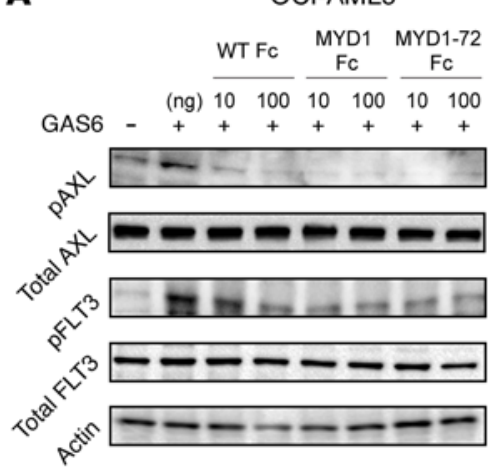

MV4:11

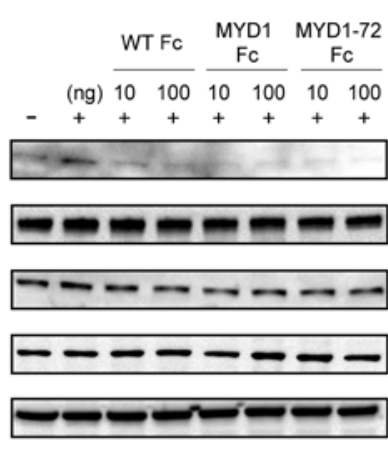

B

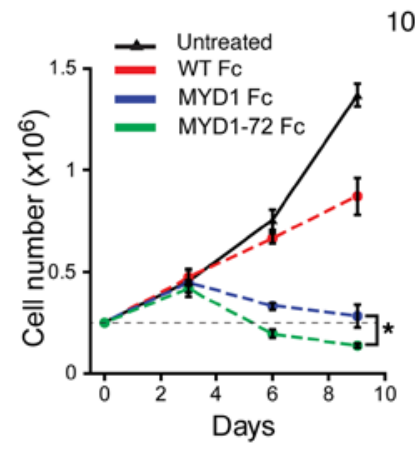

$100 \mathrm{ng} / \mathrm{ml}$

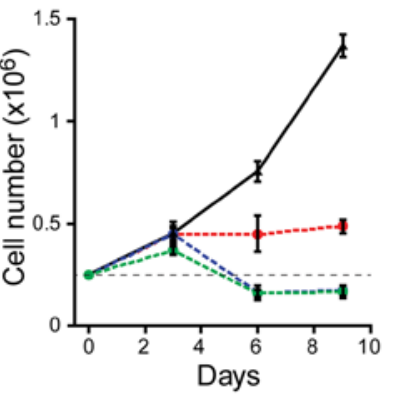

$10 \mathrm{ng} / \mathrm{ml}$

OCI-AML3
MV4:11
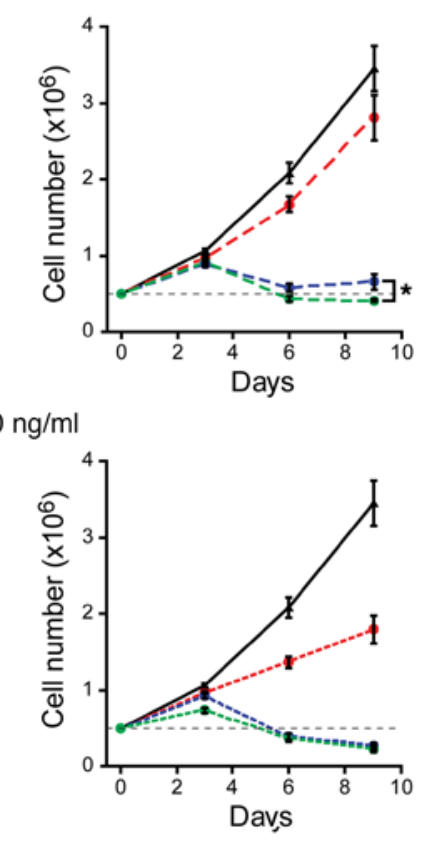

C

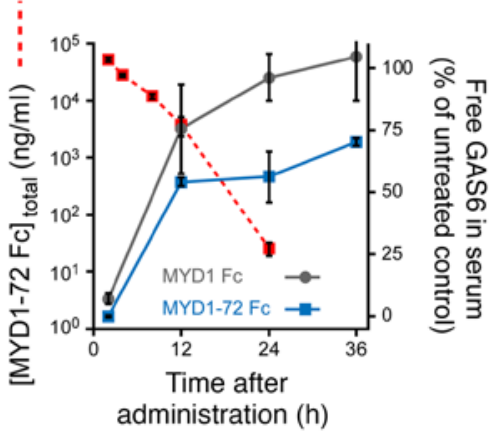

D

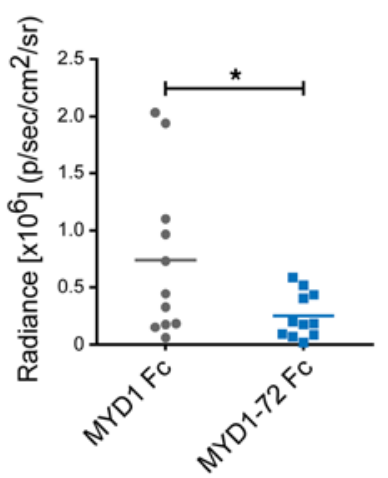

Figure 2. Superior efficacy of the second-generation AXL decoy receptor. (A) Western blots showing the reduction of AXL and FLT3 phosphorylation in AML cells when treated with the AXL decoy receptors. (B) Inhibition of cell growth and induced cytotoxicity in both OCI-AML3 and MV4:11 cells after treatment with AXL Fcs. Effects are dependent upon dosage and the affinity of the decoy receptor, but not influenced by FLT3 status. Untreated data are the same in the $100 \mathrm{ng} / \mathrm{ml}$ and $10 \mathrm{ng} / \mathrm{ml}$ graphs. (C) In vivo sequestration of CAS6 following a single $0.5 \mathrm{mg} / \mathrm{kg}$ dose of MYD1 Fc (gray) or MYD1-72 Fc (blue). The PK profile of MYD1-72 Fc following a single $1 \mathrm{mg} / \mathrm{kg}$ dose is overlaid in red. (D) Amount of lung metastases in the 4T1 breast cancer model as quantified by ex vivo bioluminescent imaging. Error bars represent mean \pm SD. $n=11$ for in vivo experiments. ${ }^{*} P<0.05$. Repeated measure ANOVA was used for measurement over time, and Student's $t$ test was used for comparing single treatment to the control.

of which are contained in MYD1 (Supplemental Table 1; supplemental material available online with this article; doi:10.1172/ JCI85610DS1). When mapped onto the GAS6/MYD1 structure (PDB: 4RA0), residue 72 was distant from the 4 mutations already contained in MYD1. Furthermore, the structural rearrangements observed on the GAS6/MYD1 structure (17) did not occur near position 72 (Supplemental Figure 1). The high frequency of A72V in the enriched pool indicated that it likely improved binding to GAS6, and its isolation in 3D space on the structure suggested it would improve the affinity of MYD1 to GAS6. To test this empirically, we recombinantly expressed an AXL Ig1 variant containing the $\mathrm{A} 72 \mathrm{~V}$ point mutation. The $\mathrm{A} 72 \mathrm{~V}$ mutation did not disrupt the overall folding of the protein (Supplemental Figure 1), and the mutant was determined to bind GAS6 with an affinity of $5.8 \mathrm{pM}$, 5 times stronger than WT AXL Ig1 (Table 1, Supplemental Figure 2, and Supplemental Table 2). Encouraged by these results, we then combined A72V with MYD1, yielding an AXL variant (MYD172) with an affinity for GAS6 of $720 \mathrm{fM}$ (Table 1). Using thermodynamic cycle analysis (Supplemental Figure 2), contributions from $\mathrm{A} 72 \mathrm{~V}$ were determined to be nearly completely additive with the preexisting mutations in MYD1, illustrating the independent nature of this mutation. When reformatted as an Fc fusion, MYD1$72 \mathrm{Fc}$ had an apparent binding affinity to human GAS6 of $93 \mathrm{fM}$, a 350-fold increase over WT AXL, and also bound more strongly to mouse GAS6 with an apparent affinity of $140 \mathrm{fM} \mathrm{().}$

Structural basis of high-affinity binding. To elucidate the structural origins underlying the affinity increase, the GAS6/MYD1-72 cocomplex was crystallized. This structure, along with the WT (PDB: 2C5D) (22) and MYD1 (PDB: 4RA0) (17) cocomplexes, use the AXL Ig1-Ig2 and GAS6 LG1-LG2 fragments to produce a 2:2 cocomplex. Despite significant effort and successful growth of crystals under several different conditions, only a low-resolution structure (3.5 ̊) was obtained (Supplemental Figure 3 and Supplemental Table 3). While the low resolution precluded detailed analysis of interresidue contacts, no significant backbone changes were observed relative to the MYD1 structure ( $\mathrm{C} \alpha$ root mean square deviation [RMSD] $0.2 \AA$ ), particularly within the regions around residue 72 and within helix A on GAS6 (Supplemental Figure 3).

In an effort to improve crystal quality, we simplified the structure by truncating AXL, complexing only MYD1-72's high-affinity Ig1 domain with GAS6, and were able to generate a high-resolution structure at $2.3 \AA$ (Figure $1 \mathrm{~B}$ and Supplemental Table 3). When compared with the GAS6/MYD1 structure (PDB: 4RAO, at 3.4A), the intermolecular contacts across the interface were similar. Importantly, analysis of the binding interface at high resolution supports previous conclusions using the GAS6/MYD1 structure. Specifical- 
A

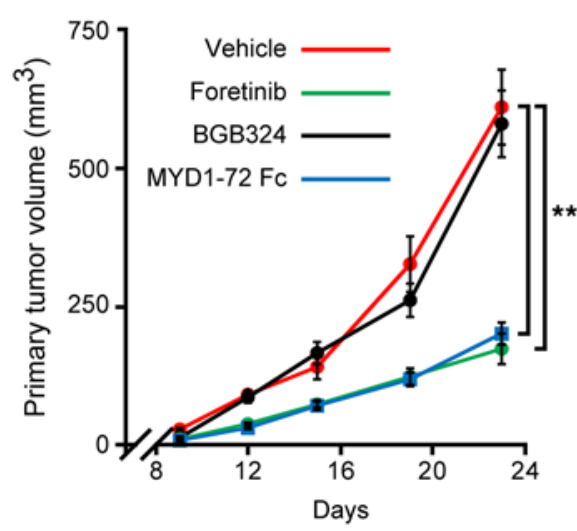

D
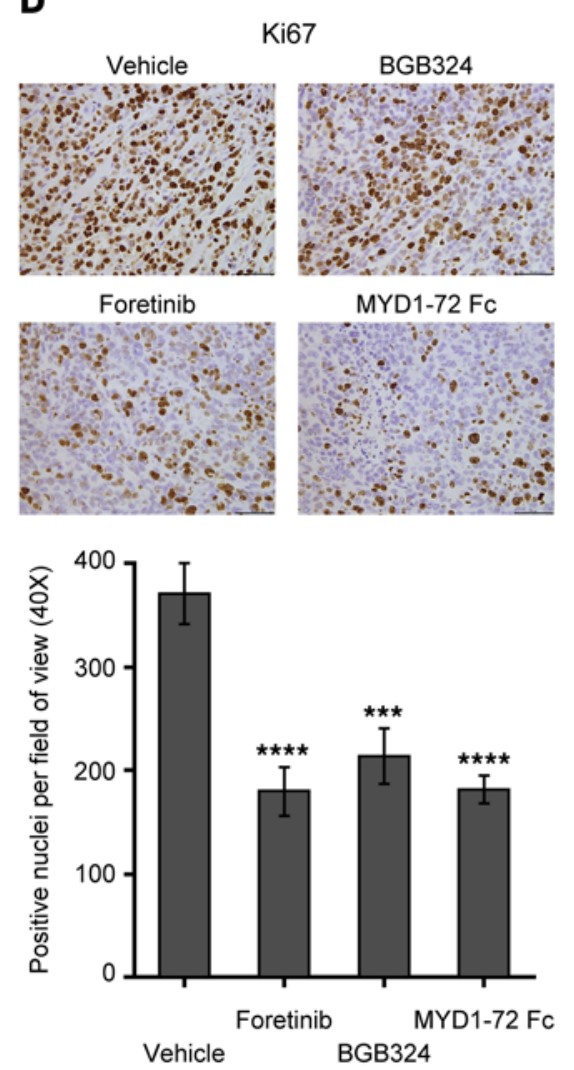

G

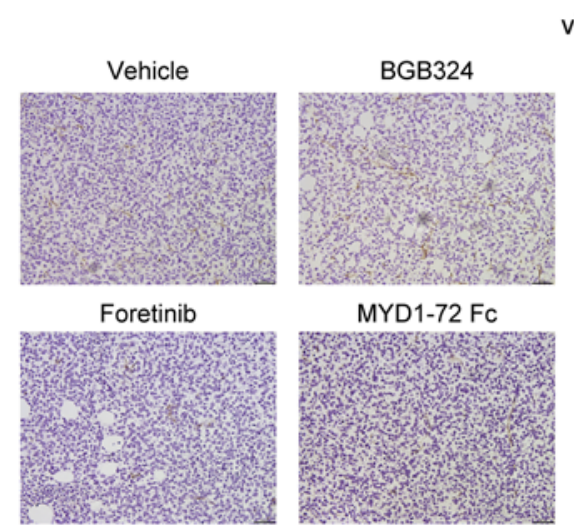

B

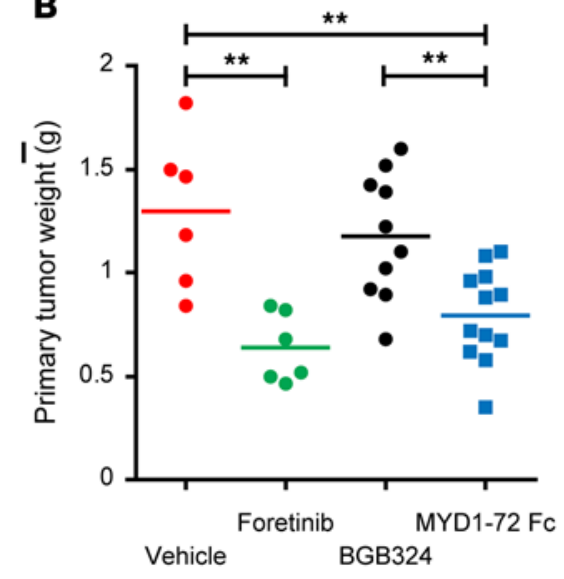

E
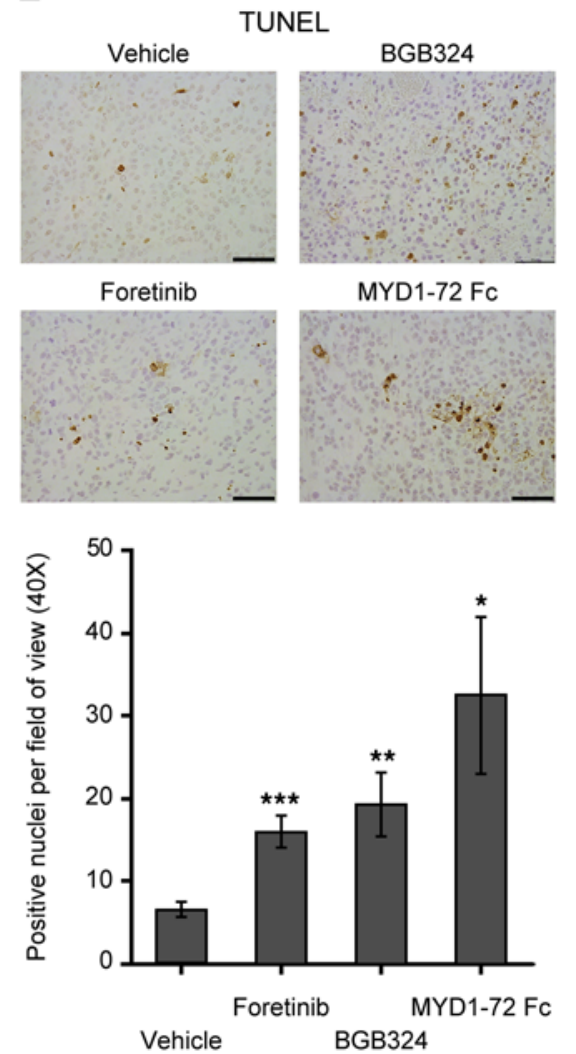

WWF

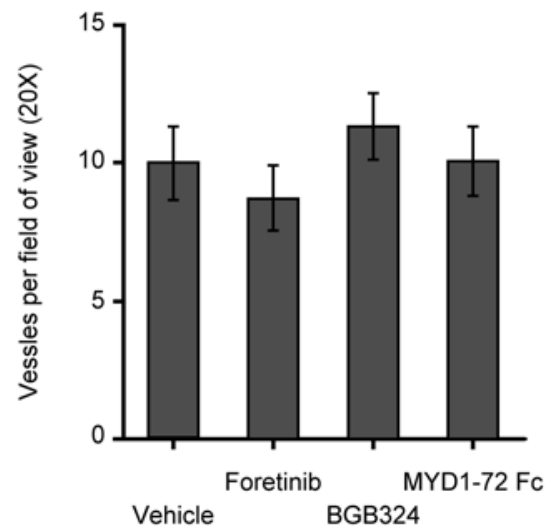

C

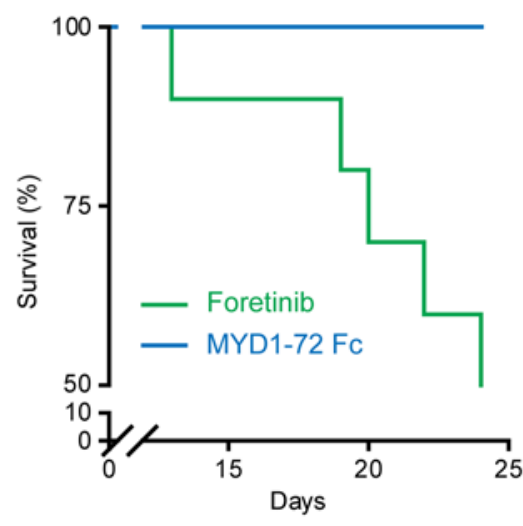

$\mathbf{F}$
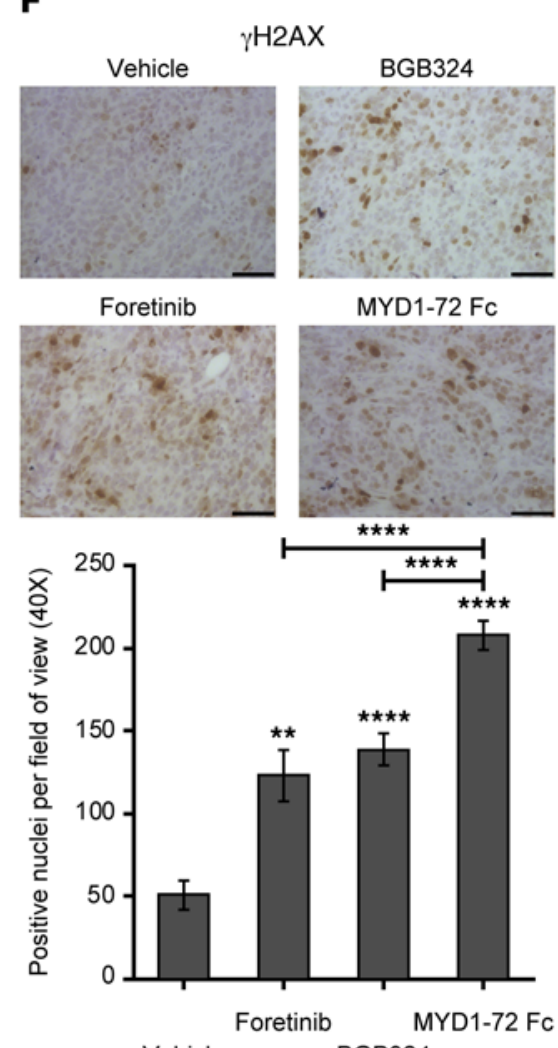

Vehicle BGB324

Figure 3. AXL inhibition reduces primary tumor growth. (A) Volume over time of orthotopically implanted primary $4 \mathrm{~T} 1$ tumors in mice treated with vehicle, foretinib, BCB324, or MYD1-72 Fc.

(B) Mass of the primary tumor at the conclusion of the study. (C) Kaplan-Meier curve for the MYD1-72 $\mathrm{Fc}-$ and foretinib-treated groups only. Toxicity from foretinib treatment required half of the mice to be prematurely removed from the study. (D-G) Representative images and matched quantification of Ki67, TUNEL, $\gamma \mathrm{H} 2 \mathrm{AX}$, and VWF staining of primary tumor tissue. Error bars represent mean \pm SD. $n=6-12 .{ }^{*} P<0.05 ;{ }^{* *} P<0.01 ;{ }^{* * *} P<0.001$;

${ }^{* * * * P}<0.0001$. Scale bars: $50 \mu \mathrm{m}$. Repeated measure ANOVA was used for measurement over time, and ANOVA with Tukey-Kramer test was used for comparing multiple treatment to each other. 
ly, the N-terminal capping of helix A on GAS6 that we reported in the MYD1 cocomplex was conserved in the MYD1-72 cocomplex. These observations serve as structural confirmation that $\mathrm{A} 72 \mathrm{~V}$ and the preexisting mutations in MYD1 act independently.

While the intermolecular contacts at the binding interface were similar between the 2 structures, substantial differences in the region around position 72 were observed. First, in the MYD172 structure, a single additional electrostatic interaction was observed on the backside of the complex within the loop containing position 72 (Figure 1C). Second, local packing within the core of AXL is markedly different (Figure 1C), as the increased volume of the valine side chain permits more efficient side-chain packing and thus a lower void volume. As a result, several van der Waals contacts are gained, further strengthening the core structure of MYD1-72 (Supplemental Table 4). Together, these 2 features likely serve as the structural basis for the improvements in affinity seen in the A72V mutant.

MYD1-72 Fc has improved anti-tumor efficacy. AXL is a known survival factor in acute myeloid leukemia (AML) (24), as cytotoxicity is seen when signaling through the receptor is antagonized. To test whether the enhanced affinity of MYD1-72 Fc would equate to increased activity, we initially characterized the effects of the decoy receptors on the in vitro growth of 2 human AML cell lines: OCI-AML3 and MV4:11. While OCI-AML3 cells are WT for FLT3, MV4:11 cells contain an internal tandem duplication (ITD) of the FLT3 receptor, resulting in constitutive activation. FLT3 ITD remains a significant clinical challenge and is associated with poor prognosis (25). Treatment with WT AXL Fc, MYD1 Fc, or MYD1-72 Fc inhibited AXL and FLT3 phosphorylation (Figure 2A), and cytotoxicity was observed in a dose-dependent manner, independent of FLT3 status (Figure 2B). Importantly, the improved affinity of MYD1-72 Fc compared with MYD1 Fc correlated with enhanced activity (Figure 2B).

To determine whether the increase in activity would translate in vivo, we evaluated how well MYD1 Fc and MYD1-72 Fc could systemically sequester endogenous GAS6. To ensure that potential improvements could be quantified, a dose of $0.5 \mathrm{mg} / \mathrm{kg}$ was used, which is the in vivo $\mathrm{IC}_{50}$ of MYD1 Fc, as previously determined using the same assay (17). Mice were administered MYD1 Fc or MYD1-72 Fc, and serum samples were obtained at time points up to 36 hours after injection. The amount of free, circulating GAS6 (i.e., not neutralized by the decoy receptor) was then quantified. Both molecules rapidly eliminated free GAS6 upon administration, though MYD1-72 Fc suppressed GAS6 levels longer than MYD1 Fc (Figure 2C), highlighting its improved pharmacodynamic (PD) profile. These improvements were not due to differences in the clearance rates of the molecules, as the PK profile of MYD1-72 Fc was similar to what was previously reported for MYD1 Fc (Figure 2C).

To directly study the consequences of MYD1-72 Fc's improved PD, we used both decoy receptors as treatments in the 4T1-luciferase breast cancer model of metastasis. In this model, cells orthotopically implanted in the mammary fat pad generate primary masses that metastasize to the lungs. Four days after tumor implantation, mice were treated with either MYD1 Fc or MYD1-72 Fc at the suboptimal dose of $0.5 \mathrm{mg} / \mathrm{kg}$. After 3 weeks of treatment, mice receiving MYD1-72 Fc had sig- nificantly less metastatic disease than those treated with MYD1 Fc (Figure 2D). Collectively, these studies demonstrate the superior efficacy of our second-generation molecule, further emphasizing the important correlation of the decoy receptor's affinity to therapeutic efficacy.

Comparison of MYD1-72 FC with clinical AXL tyrosine kinase inhibitors. There are currently no FDA-approved drugs targeting AXL, though several small molecule kinase inhibitors are undergoing clinical trials. BerGenBio's BGB324 (previously Rigel's R428) is the most advanced of these compounds and the first to be developed prospectively as an AXL inhibitor (26). Given the positive clinical results seen thus far with BGB324, we hypothesized that it would serve as a good benchmark of comparison for MYD1-72 Fc. Furthermore, since tyrosine kinase inhibitors (TKIs) and biologics are fundamentally very different, as are their mechanisms of action, a direct comparison would provide valuable insights into the potential of distinct therapeutic intervention strategies.

We therefore compared the antitumor effects of MYD1-72 Fc to BGB324 and another TKI with significant activity against AXL, foretinib (27), in the 4T1-luciferase model. Cells were implanted orthotopically in the mammary fat pad, and primary tumors were allowed to establish prior to treatment. Mice were then randomized into 1 of 4 treatment groups: saline, MYD1-72 Fc daily at $1 \mathrm{mg} /$ $\mathrm{kg}$, BGB324 twice daily at $12.5 \mathrm{mg} / \mathrm{kg}$, or foretinib twice daily at $12.5 \mathrm{mg} / \mathrm{kg}$. This dose for the TKIs was chosen as significant activity has been previously reported at equivalent concentrations (26, 28). Mice were treated for 3 weeks or until they showed signs of morbidity, at which point they were removed from the study.

Both MYD1-72 Fc and foretinib significantly reduced the size of primary tumors compared with control mice, while BGB324 showed little effects (Figure 3, A and B). This was notable because, with the exception of AML, AXL has historically been a driving force of metastatic disease, showing little effect on cellular growth in vitro or primary tumor growth in vivo (8). Though antitumor efficacy was seen in the foretinib-treated group, substantial toxicity was also present, requiring half of the mice to be sacrificed prior to the study endpoint (Figure 3C). Immunohistochemistry was performed on sections of primary tumor from each treatment group to study the mechanism underlying the reduction in growth. Staining for the proliferation marker Ki67 revealed all treatment groups had significantly less proliferation within the primary tumor compared with control animals (Figure 3D). Complimenting this, there

\section{Table 1. GAS6-binding parameters}

\begin{tabular}{|c|c|c|c|c|}
\hline & $\begin{array}{l}K_{D}(\mathrm{fM}) \\
\text { hGAS6 }\end{array}$ & $\begin{array}{c}K_{o n} \\
\left(10^{7} M^{-1} s^{-1}\right)\end{array}$ & $\begin{array}{c}K_{\text {off }} \\
\left(10^{-5} s^{-1}\right)\end{array}$ & $\begin{array}{l}K_{D}(\mathrm{fM}) \\
\text { mGAS6 }\end{array}$ \\
\hline WT AXL $\lg 1^{B}$ & 33,000 & 2.1 & 70 & ND \\
\hline MYD1 lg1 ${ }^{B}$ & 2,700 & 1.6 & 4.0 & ND \\
\hline A72V Ig1 & 5,800 & 1.9 & 11.0 & ND \\
\hline MYD1-72 Ig1 & 720 & 1.7 & 1.2 & ND \\
\hline MYD1 Fc ${ }^{B}$ & $420^{A}$ & 2.3 & 1.0 & 1,100 \\
\hline MYD1-72 Fc & $93^{A}$ & 2.7 & 0.25 & 140 \\
\hline
\end{tabular}

${ }^{A}$ Values that are apparent affinities, not affinities. ${ }^{B}$ Previously reported values in ref. 17. ND, not detectable. 
A

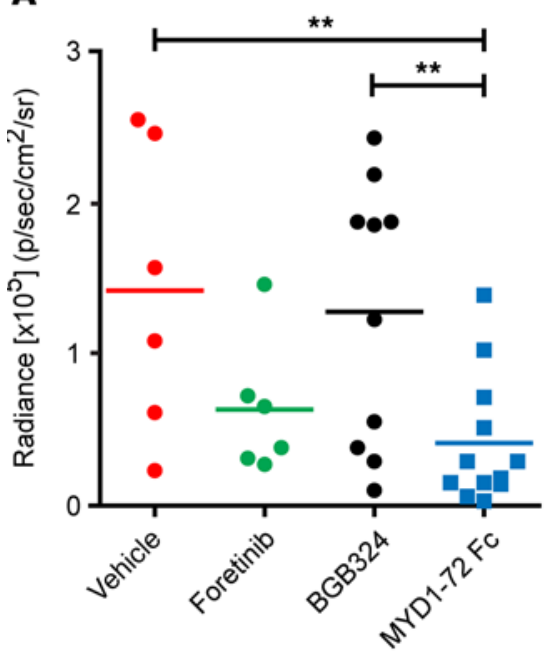

C

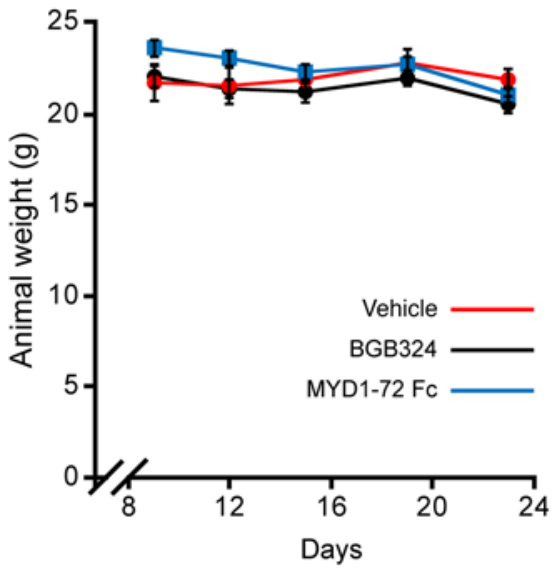

B

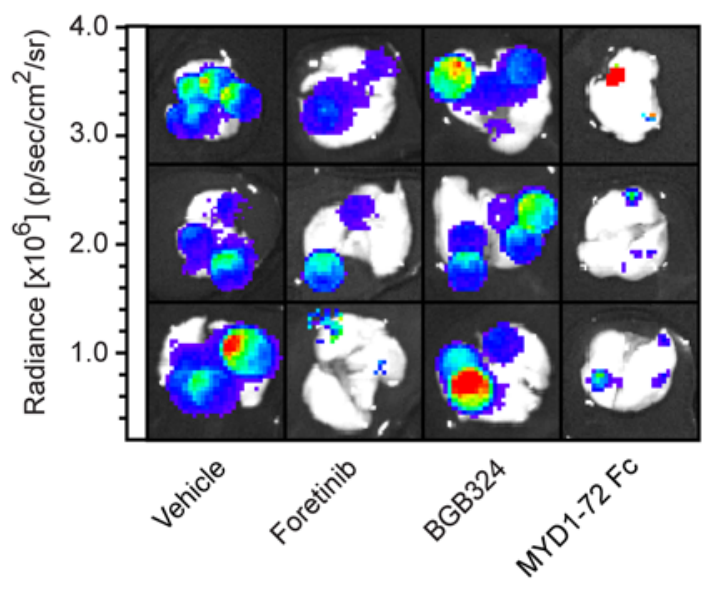

D

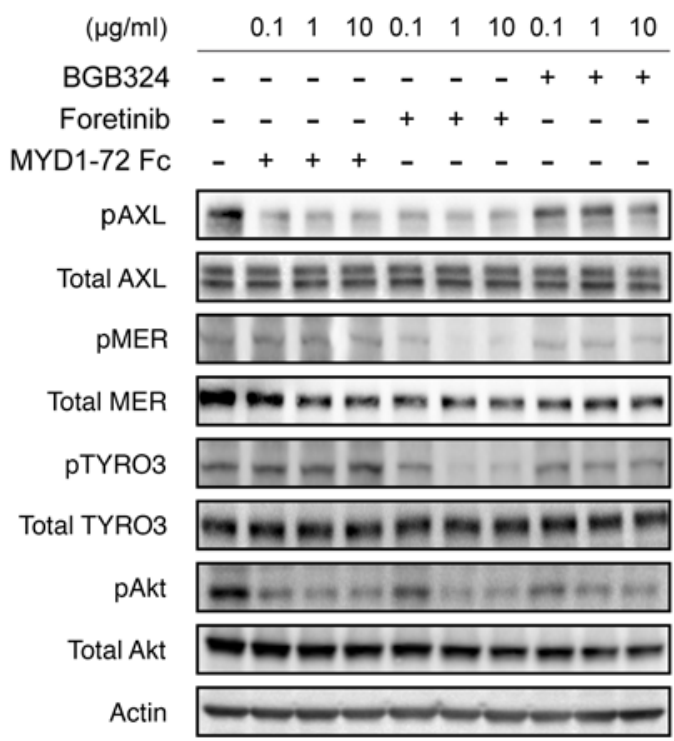

Figure 4. Inhibition of AXL decreases metastatic tumor burden. (A) Lung metastases in the 4T1 model, quantified ex vivo by bioluminescent imaging. (B) Representative bioluminescent images of whole lungs from mice in each treatment group. (C) Average animal weight in each treatment group over the course of the study. Foretinib was omitted as animals were removed throughout the study. (D) Western blot analysis of OVCAR8 cells after 4-hour treatment with BCB324, foretinib, or MYD1-72 Fc. Activation of all 3 TAM receptors as well as downstream Akt signaling was assayed. Error bars represent mean \pm SEM. $n=6-12$. ${ }^{*} P<0.01$ Repeated measure ANOVA were used for measurement over time, and ANOVA with Tukey-Kramer test was used for comparing multiple treatments to each other. was also more intratumoral apoptosis in the treatment groups (Figure 3E). To further understand the increased apoptosis rate, we stained for $\gamma \mathrm{H} 2 \mathrm{AX}$, a marker of DNA double-strand breaks. All treatment groups showed elevated levels of $\gamma \mathrm{H} 2 \mathrm{AX}$ compared with controls; however, tissue samples from MYD1-72 Fc-treated animals had significantly more $\gamma \mathrm{H} 2 \mathrm{AX}$-positive cells compared with all other treatment groups (Figure 3F). While AXL has been linked to VEGF signaling and endothelial cells $(29,30)$, no changes in vessel density were observed across the groups (Figure $3 G$ ).

MYD1-72 Fc and foretinib also demonstrated activity against metastatic disease, as seen by a $71 \%$ and $55 \%$ reduction in lung metastases, respectively, compared with vehicle-treated mice (Figure 4, A and B). In contrast, BGB324 had a modest, 10\%, decrease that was not significantly different than that in control animals. Throughout the study, both MYD1-72 Fc and BGB324 were well tolerated with no visible signs of toxicity, as animal weight remained consistent across treatment groups (Figure 4C).

To further understand these differences among MYD1-72 Fc, foretinib, and BGB324, we examined changes in the phosphory- lation of AXL, MER, TYRO3, and downstream Akt signaling in vitro upon treatment with each. MYD1-72 Fc significantly reduced pAXL and pAkt levels, while pMER and pTYRO3 remained relatively unchanged (Figure 4D). These data indicate that MYD1-72 $\mathrm{Fc}$ is specific at attenuating the AXL-signaling cascade, achieving significant efficacy without noticeable off-target effects. In contrast, BGB324 treatment resulted in short-term inhibition of pAXL, but increased pAXL levels at later time points (Figure 4D and Supplemental Figure 4). While efficacious, the promiscuity of foretinib, which indiscriminately inhibited all TAM receptors (Figure 4D and Supplemental Figure 4), was likely responsible for the significant toxicity observed.

Collectively, these data demonstrate that MYD1-72 Fc compares favorably to anti-AXL TKIs currently being evaluated in the clinic, both in terms of safety and efficacy. Specifically, MYD1-72 Fc recapitulates the efficacy of foretinib without the aforementioned toxicity, while achieving greater antitumor effects than BGB324.

AXL signaling augments the DNA-damage response. AXL signaling is known to be a critical driver of tumor progression and drug 
resistance $(12,31)$, leading to interest in combining AXL inhibitors with other targeted therapies. Our data suggest that inhibiting AXL induces a DNA-damage response, as demonstrated by elevated $\gamma \mathrm{H} 2 \mathrm{AX}$ levels in the primary tumors of the 4T1 study (Figure 3F). To further interrogate the link between AXL and the DNA-damage response, we first performed immunofluorescence staining to detect $\gamma \mathrm{H} 2 \mathrm{AX}$ foci in ovarian cancer cells treated with $0.1 \mu \mathrm{g}$ or $10 \mu \mathrm{g}$ of MYD1-72 Fc. While cells treated with MYD1-72 $\mathrm{Fc}$ in complete media showed no changes across treatments (Figure $5 \mathrm{~A}$ ), treatment done under serum-limited conditions resulted in a significant increase in the number of $\gamma \mathrm{H} 2 \mathrm{AX}$ foci compared with untreated controls (Figure 5A), suggesting that a DNAdamage response is activated upon inhibition of AXL signaling.

To further understand this relationship, we performed a reverse phase protein array (RPPA) on shAXL cell lines and WT cell lines treated with MYD1-72 Fc (Supplemental Figure 6). We found Akt, mTor, and P70SK6 to be differentially expressed in all data sets (Supplemental Figure 6-8), demonstrating the fidelity of both assay and samples, as these downstream effectors are known to be controlled in part by AXL signaling (32). Interestingly, key components of the DNA-damage response (XRCC1/ CHK2) as well as some proapoptotic members of the BCL-2 pathway (BAX/BID) were highly upregulated in the shAXL cell lines (Supplemental Figure 7) and when WT cells were treated with MYD1-72 Fc continuously for 7 days in low serum (1\% FBS) (Supplemental Figure 7 and 9). In addition, we performed Western blot analysis to examine expression levels of classical DNA-damage response components, including total and phosphorylated ATM, ATR, CHK1, CHK2, and RPA32 (Figure 5B), at 4 time points after MYD1-72 treatment. We saw induction of pATM and pATR at early time points, followed by increased phosphorylation of both CHK1 and CHK2. Elevated pRPA32 levels were also observed (as shown by the doublet band on the total RPA32 blot), suggesting replication stress. These data suggest that the loss of AXL signaling can modulate DNA-damage response signaling in tumor cells under stress conditions such as nutrient deprivation.

To interrogate the nature of the DNA-damage response, we immunofluorescently stained cells treated with MYD1-72 Fc for 53BP1 and RAD51 foci. Under serum-limited conditions, treatment with MYD1-72 Fc increased the numbers of both 53BP1 and RAD51 foci (Supplemental Figure 5), further supporting our hypothesis that loss of AXL signaling promotes a DNA-damage response. Intrigued by the RPA phosphorylation observed following AXL inhibition (Figure 5B), we performed EdU labeling and costaining of cells for $\gamma \mathrm{H} 2 \mathrm{AX}$ (Figure 5C). These experiments allow a better understanding of whether the $\gamma \mathrm{H} 2 \mathrm{AX}$ signaling previously observed following AXL inhibition was occurring in EdU-positive/S phase cells, suggesting a link between AXL inhibition and replication stress. Figure 5D shows the percentage of $\gamma \mathrm{H} 2 \mathrm{AX}$-positive cells upon treatment with MYD1-72 Fc. This result supports the previous observation that treatment with MYD1-72 Fc induces $\gamma \mathrm{H} 2 \mathrm{AX}$ signal in cancer cells. There was a minor difference in the number of $S$ phase cells between control and MYD-72 Fc-treated groups that is unlikely to alone account for the difference in distribution of the $\gamma \mathrm{H} 2 \mathrm{AX}$ signal (Figure 5E). Interestingly, we observed that in the MYD-72 Fc-treated group, $85 \%$ of cells that were $\gamma \mathrm{H} 2 \mathrm{AX}$ positive were also in $\mathrm{S}$ phase/EdU positive (Fig- ure $5 \mathrm{~F}$ ), while only $7 \%$ of non-S phase cells (EdU negative) were positive for $\gamma \mathrm{H} 2 \mathrm{AX}$ (Supplemental Figure 5).

Furthermore, cells treated with hydroxyurea $(\mathrm{Hu})$ in the presence and absence of MYD1-72 Fc showed comparable numbers of $\gamma \mathrm{H} 2 \mathrm{AX}$-positive cells, and no additive effect was observed when the 2 compounds were used in combination (Supplemental Figure 5). Together, these results suggest that AXL inhibition may contribute to replication stress, resulting in increasing DNA-damage signaling.

That these effects were observed only under serum-limited conditions is further indication that GAS6/AXL signaling provides a cytoprotective effect for tumor cells placed under stress, such as growth factor deprivation. These results provide the rationale for combining AXL inhibitors and DNA-damaging agents, such as radiation and chemotherapy, to enhance their therapeutic index.

Inhibiting AXL improves the standard of care. Clinical management of ovarian cancer remains a challenge, as patients often present with advanced metastatic disease at the time of diagnosis. Treatment for these patients is limited and entails surgical debulking followed by combination chemotherapy; however, tumor response rates remain poor (33). Coincidently, ovarian cancer represents an ideal setting to test the combination of AXL inhibitors and DNA-damaging agents, as patients are in critical need of new treatment options that can enhance the effects of standard-of-care chemotherapy.

We therefore treated ovarian cancer cells with MYD1-72 Fc either alone or in combination with doxorubicin in vitro and assessed levels of DNA damage by staining the cells for $\gamma \mathrm{H} 2 \mathrm{AX}$ foci. Cells treated with MYD1-72 Fc alone showed a significant increase in the number of $\gamma \mathrm{H} 2 \mathrm{AX}$ foci compared with untreated controls; however, $\gamma \mathrm{H} 2 \mathrm{AX}$ levels were further elevated when doxorubicin was also present (Figure 5A). These data further support the link between inhibition of AXL signaling and the DNA-damage response and suggest that a synergistic effect can be achieved when anti-AXL therapies are combined with cytotoxic chemotherapy.

To see whether these results would translate in vivo, we evaluated the efficacy of MYD1-72 Fc alone and in combination with doxorubicin in 2 models of human ovarian cancer, the OVCAR8 and skov3.ip models. For both models, cells were injected i.p. where they were allowed to establish for 1 week prior to treatment. At this time, several mice were sacrificed at random to ensure engraftment of diffuse metastatic disease, a hallmark of the human condition. Mice were then randomized into 1 of 4 treatment groups: saline, MYD1-72 Fc at $1 \mathrm{mg} / \mathrm{kg}$ daily, doxorubicin at $2 \mathrm{mg} / \mathrm{kg}$ twice weekly, or a combination of MYD1-72 Fc and doxorubicin. After 3 weeks of treatment, mice were sacrificed and tumor burden was quantified by counting the number of visible lesions as well as excising and weighing all diseased tissue.

In the OVCAR8 model, MYD1-72 Fc had significant antitumor effects, reducing tumor burden by $95 \%$ as a single agent (Figure 6A). While similar effects were obtained using doxorubicin alone, combination treatment resulted in nearly undetectable levels of disease. Each mouse receiving both therapies had on average 2 and at most 3 macroscopic metastases, while the mean number in the control group was in excess of 750 (Figure 6A, inlaid graph). A similar degree of diminishment was observed when comparing cumulative metastatic mass (Figure 6A). 
A

$0.1 \%$ serum

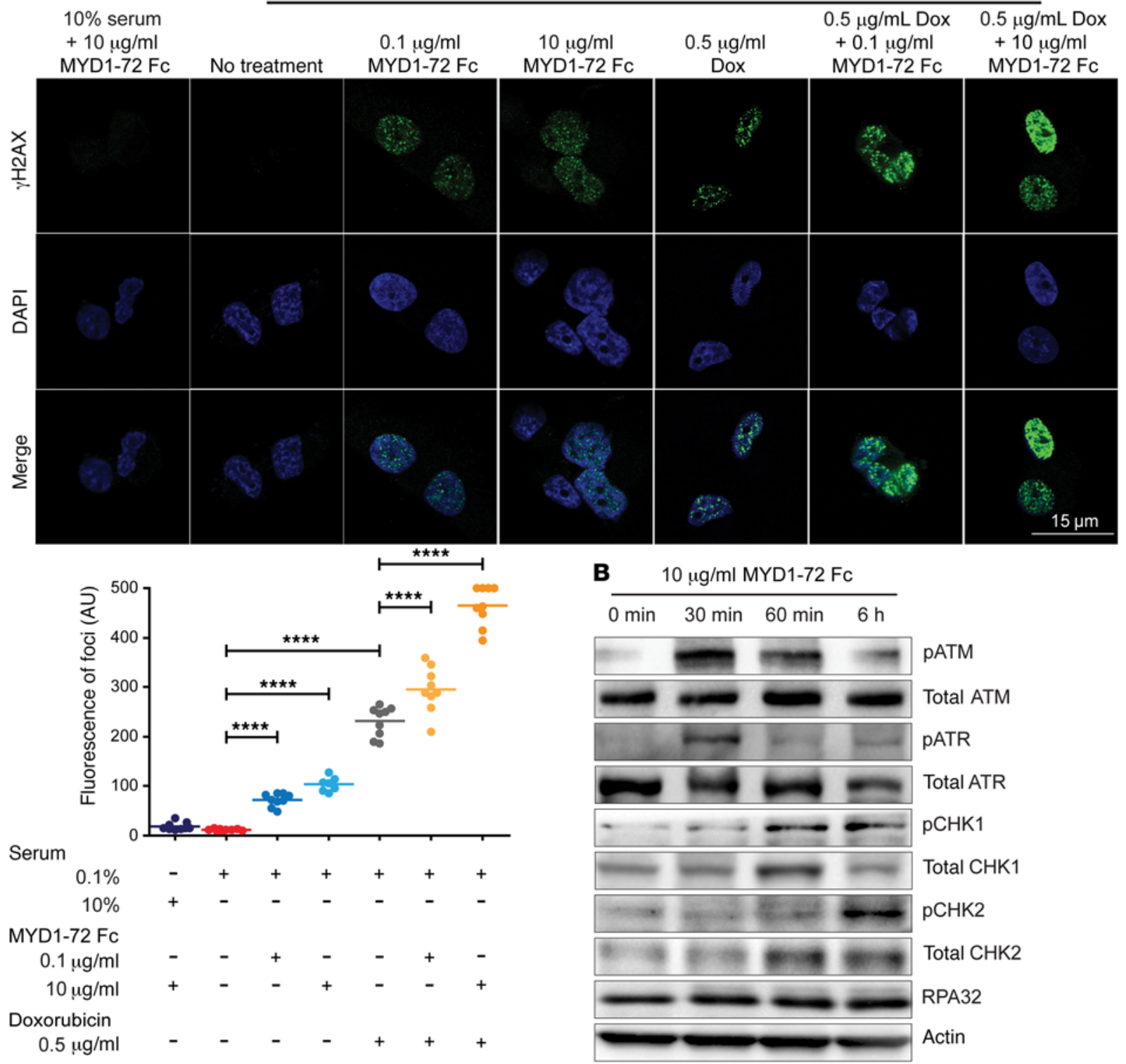

C

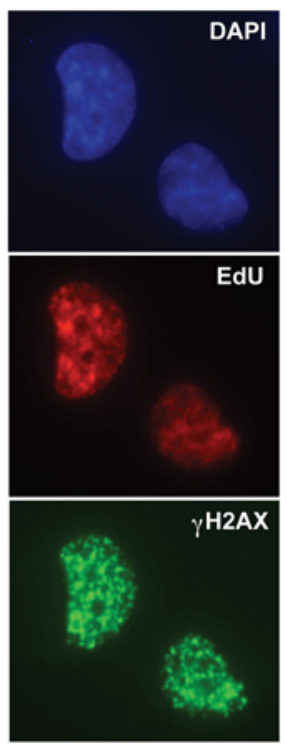

D

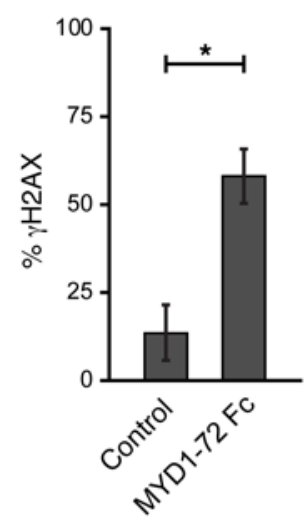

E

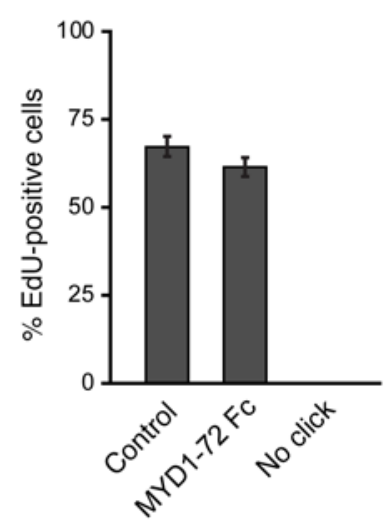

$\mathbf{F}$

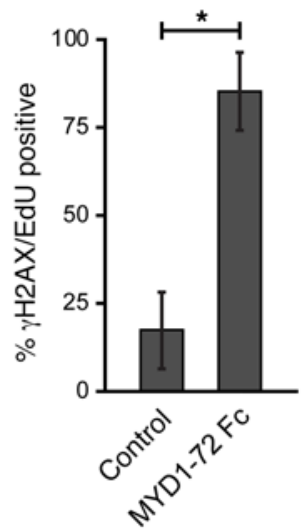


Figure 5. Treatment with MYD1-72 induces DNA-damage response during cell S phase. (A) Immunofluorescence staining of $\gamma \mathrm{H} 2 \mathrm{AX}$ foci formation in ovarian cancer cells treated with MYD1-72 Fc alone or in combination with doxorubicin. $n=7-9$. (B) Western blot analysis was carried out to examine changes in the phosphorylation of ATM, ATR, CHK1, CHK2, and RPA32 in ovarian cancer cells cultured in low serum (0.1\% FBS) after treatment with MYD1-72 Fc. (C) Representative images of EdU- and $\gamma \mathrm{H} 2 \mathrm{AX}$-positive cells and (D) quantification of $\gamma \mathrm{H} 2 \mathrm{AX}$-positive cells in MYD1-72 Fc vs. control treated cells. The differences in the number of $\mathrm{S}$ phase cells (EdU positive) between control and MYD1-72 Fc-treated groups are shown. No click refers to the negative control for EdU staining (E). The percentage of $\gamma \mathrm{H} 2 \mathrm{AX}$-positive cells that are in $\mathrm{S}$ phase (EdU positive) between control and MYD1-72 Fc-treated group is shown in $\mathbf{F}$. $n=3$. ${ }^{*} P<0.05 ;{ }^{* * *} P<0.0001$. Scale bars: $15 \mu \mathrm{m}$. ANOVA with Tukey-Kramer test was used for comparing multiple treatments to each other, and Student's $t$ test was used for comparing single treatments to the control.

Comparatively, the skov3.ip model was more aggressive, with mice in the control group having nearly 3 times as much tumor tissue, by weight, at the conclusion of the study as in the OVCAR8 study (Figure 6B). Under these conditions, using the number of nodules as a measure of tumor burden became misleading, as lesions grew into one another, resulting in a smaller number of larger metastases. Even in this more advanced setting, MYD1-72 Fc and doxorubicin had significant antitumor activity, decreasing tumor burden by $51 \%$ and $91 \%$, respectively (Figure 6B). The combination of these 2 agents was once again effective, as animals in the combination group had, on average, $99 \%$ less tumor by weight than controls. Furthermore, within this group, 3 out of 10 animals were completely cured with no evidence of disease (Figure 6B, inlaid graphs). Together, these 2 studies support the in vitro findings that AXL inhibition modulates the DNA-damage response and demonstrate that antagonizing the GAS6/AXL signaling axis can be leveraged to improve the therapeutic index of chemotherapy.

Successful inhibition of AXL carries with it little risk of on-target toxicity, as seen in the AXL-knockout mouse (14). However, broad inhibition of the TAM family can lead to severe toxicities. For example, autoimmune diseases are common in the TAM triple-knockout mouse (14) and disruption of the retinal pigmented epithelium (RPE) can lead to blindness when signaling through MER is abrogated (34). Furthermore, concurrent inhibition of AXL and MER has been shown to enhance tumor growth in certain types of cancer (35). To ensure the decoy receptors were specifically targeting AXL in vivo, we performed histology to assess the integrity of the RPE in mice from the skov3.ip experiment. Across all treatment groups, the RPE was healthy and normal, indicating that requisite signaling through other TAM family members, specifically MER, is preserved (Figure 6C). Additionally, histological analysis on the liver, lung, and kidney from these animals showed no histological abnormalities across treatment groups, indicating a lack of gross toxicity (Figure 6C).

Combination treatment improves overall survival of pancreatic cancer. Similar to recurrent ovarian cancer, pancreatic cancer represents a largely intractable clinical challenge. The primary tumor location makes early detection improbable, and patients often present with advanced disease, as illustrated by 5 -year survival rates of around 6\% (36). Even when surgery is feasible, adjuvant chemotherapies are nearly ubiquitously administered in the form of the pyrimidine antagonists gemcitabine or fluorouracil (5-FU) (37). Pancreatic cancer thus represents an additional clinical setting in which improved therapies are desperately needed and standard treatment includes DNA-damaging agents.

To examine whether the synergistic effects seen in the ovarian models were indicative of a general phenomenon broadly applicable to clinical oncology, MYD1-72 Fc was tested alone and in com- bination with gemcitabine in an orthotopic model of murine pancreatic cancer. LMP cells derived from the KRAS/p53 metastatic mouse model (38) were implanted s.c. into the flanks of mice and allowed to grow until they reached approximately $500 \mathrm{~mm}^{3}$ in size. To establish orthotopic tumors, mice harboring s.c. tumors were sacrificed and tumors were isolated and cut into small fragments. Laparotomies were then performed, and a tumor fragment was secured to the tail of the pancreas. Four days after engraftment, mice were randomized into 1 of 4 treatment groups: saline, MYD1$72 \mathrm{Fc}$ at $1 \mathrm{mg} / \mathrm{kg}$ daily, gemcitabine at $100 \mathrm{mg} / \mathrm{kg}$ twice weekly, or a combination of MYD1-72 Fc and gemcitabine. Dosing continued until a mouse showed significant signs of morbidity, at which time it was removed from the study.

Direct engraftment of tumor tissue to the pancreas yielded a rapidly progressing primary tumor, with a median survival in the control group of 17 days (Figure 7 and Table 2). As single agents, MYD1-72 Fc showed no activity with a median survival of seventeen days, while gemcitabine doubled median survival to 35 days. As in the ovarian models, combining MYD1-72 Fc and chemotherapy showed significantly greater efficacy over either therapy alone, as median survival was tripled to 57 days (Figure 7 and Table 2). Across treatment groups, animals succumbed to large primary tumor masses rather than diffuse metastatic disease. To determine whether increased sensitivity to DNA damage was the mechanism driving the effects seen in the combination group, immunohistochemistry was performed on primary tumor tissue samples. Ki67 staining showed a small, albeit significant decrease in proliferation within the gemcitabine-treated group (Figure 8A), while vessel density remained unchanged (Figure $8 \mathrm{~B}$ ). Intratumoral apoptosis was significantly increased in all treatment groups compared with controls, particularly in those animals administered MYD1-72 Fc despite the fact that the decoy receptor alone demonstrated negligible effects on overall survival (Figure 8C). Most notably, although MYD1-72 Fc treatment had a small effect on DNA damage, the combination of MYD1-72 Fc and gemcitabine significantly increased the amount of $\gamma \mathrm{H} 2 \mathrm{AX}$ staining compared with all other groups (Figure 8D). These results strengthen the link between AXL inhibition and the DNA-damage response and, in combination with the data from the $4 \mathrm{~T} 1$ and ovarian cancer models, highlight it as an important mechanism across cancer types. Furthermore, along with the ovarian cancer studies, these data convincingly demonstrate that this relationship can be exploited to achieve meaningful improvements in overall response rates over what can be realized by current clinical standards of care.

\section{Discussion}

As our understanding of the molecular basis of cancer has improved, a number of dysregulated signaling pathways respon- 
A

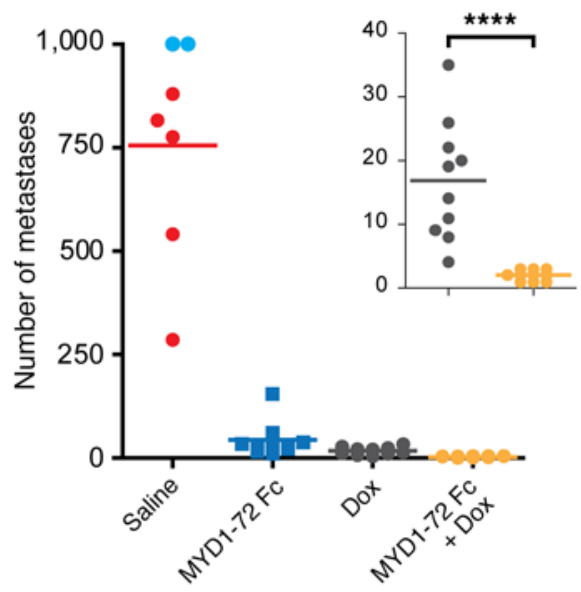

B

skov3.ip

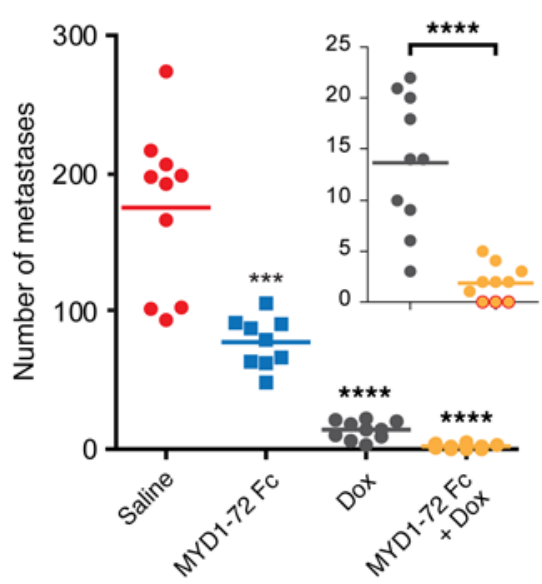

OVCAR8

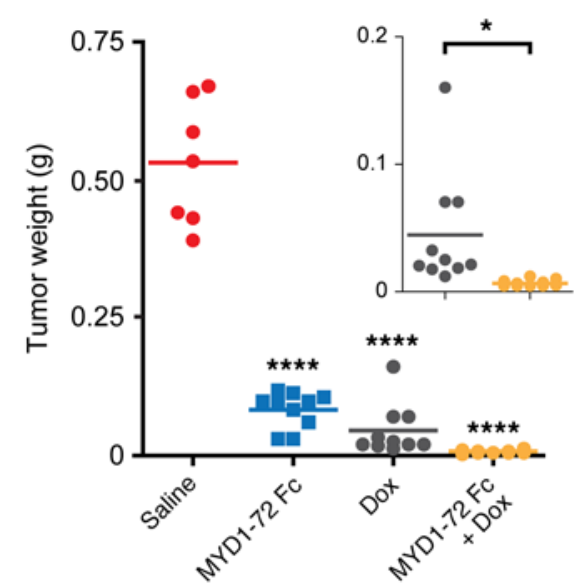

C

C

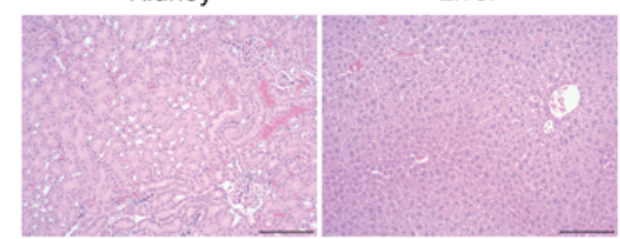

등

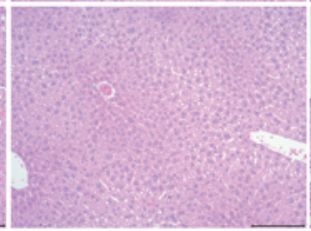

i

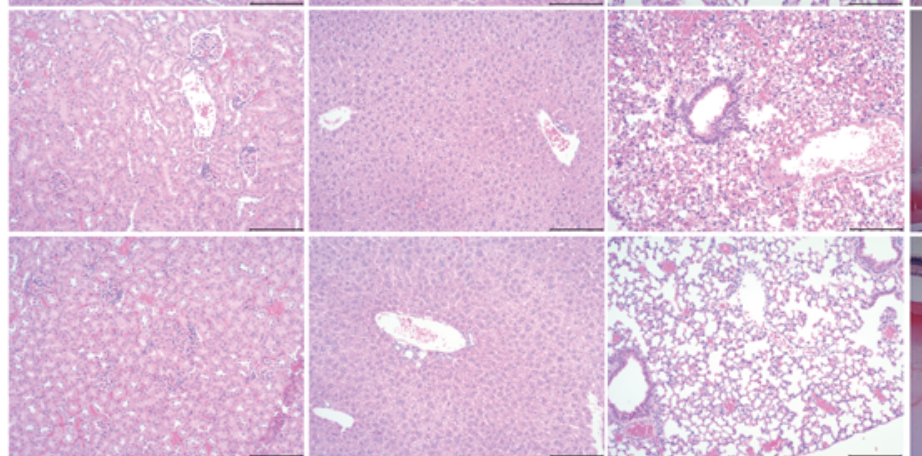

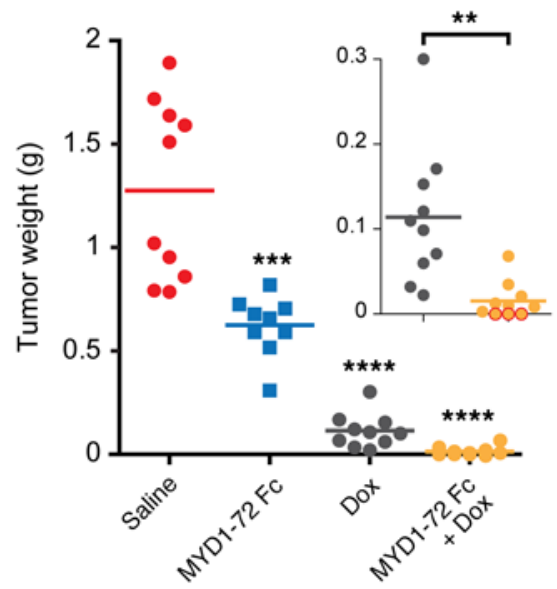

Lung

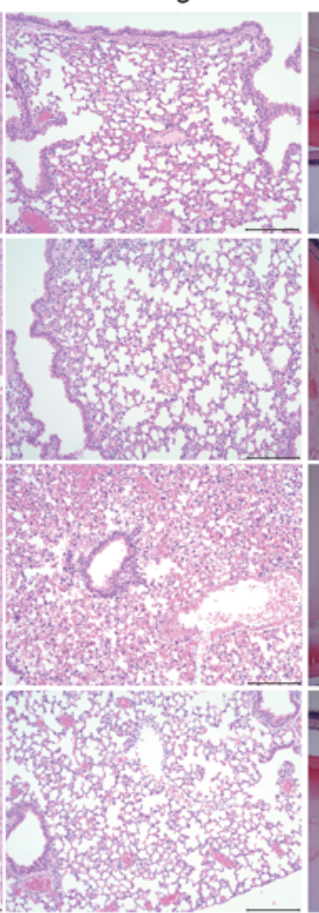

Eye (RPE)

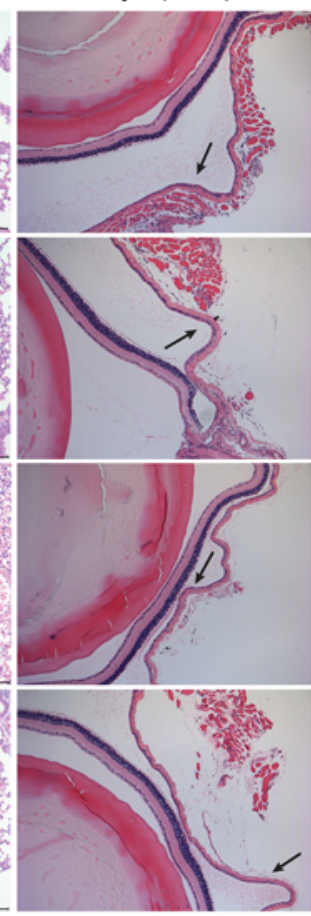

Figure 6. MYD1-72 Fc enhances the effects of chemotherapy in ovarian cancer. Inhibition of metastasis in the OVCAR8 (A) and SKOV3.ip (B) ovarian cancer models as measured by number of metastases and overall tumor weight. Blue data points represent mice estimated to have over 1,000 metastases, and red outlined data points represent mice with no evidence of disease. Inlaid graphs are expanded views of a subset of the complete data set to highlight differences between treatment groups. The eyes, liver, lungs, and kidneys of mice in the OVCAR8 study (C) were analyzed for histological signs of toxicity. Treatment with MYD1-72 Fc did not result in ocular toxicity; as the integrity of the RPE (arrows) was maintained, no histological abnormalities were present across treatment groups. Representative images are shown from each treatment group. $n=7-10 .{ }^{*} P<0.05$; ${ }^{* *} P<0.01$; ${ }^{* *} P<0.001 ;{ }^{* * *} P<0.0001$. Scale bars: $50 \mu \mathrm{m}$. Dox, doxorubicin. ANOVA with Tukey-Kramer test was used for comparing multiple treatments to each other. 
sible for driving disease progression have been identified. Efforts have been made to exploit these pathways as targets for therapeutic intervention, with the expectation that drugs capable of modulating them would deliver previously unachievable efficacy. In practice, however, the genetic instability and hypermutation rates of cancer, coupled with the redundancy often built in to biological systems, have undermined the importance of singular targets. In the presence of this confluence of factors, resistant mutations arise and compensatory signaling pathways become upregulated, limiting the utility of specific inhibitors. As an illustration, clinical trials for some new molecular entities prospectively set progression-free survival as the primary endpoint (39), highlighting the difficulty in achieving meaningful advancements in overall survival. These observations suggest that, while the development of new targets is critical, priority should be given to those that have the potential to act in synergy with, and increase the therapeutic index of, established treatment modalities. The AXL receptor fits this description. AXL initially attracted attention because of the fundamental roles it plays in driving tumor progression and metastatic dissemination, but more recently, relationships between the receptor and other disease pathways have emerged. The intersection of these two features has thus made AXL an ideal oncology target.

There is a critical need for the development and characterization of anti-AXL therapeutics. To this end, a pipeline of AXL antagonists has been generated that largely comprises small molecule kinase inhibitors (40). The paucity of biologics under clinical development can be attributed to the comparatively poor binding affinity of the mAbs described to date, as the strength of the native GAS6/AXL interaction is orders of magnitude stronger than each $\mathrm{mAb}$. As an example, several generations of anti-AXL Abs were developed with affinities to the receptor in the nanomolar range (7, 41). When tested in preclinical models, modest antitumor effects were seen, hinting at the value of the target while highlighting the difficulties inherent to inhibiting AXL.

We have previously demonstrated that the use of an engineered AXL decoy receptor is an effective way to exploit the native interaction itself in order to overcome this affinity barrier. Here, by developing a second-generation decoy receptor, we further validate this approach and more clearly define the relationship between the affinity of our AXL antagonists and antitumor effects achieved in vivo. This reengineered molecule, MYD1-72, combines an additional beneficial mutation, allowing it to attain a subpicomolar affinity to GAS6. Comparisons of the first- and secondgeneration receptors demonstrated that this improved binding affinity was critical for achieving optimal efficacy. Importantly, at $93 \mathrm{fM}$, the apparent affinity of the final MYD1-72 Fc construct represents one of the strongest protein-protein interactions reported. An additional feature of MYD1-72 is that it binds strongly to both mouse and human GAS6. The original MYD1 showed no increase in binding to mouse GAS6, preventing it from effectively neutralizing endogenous mGAS6 in preclinical models. By binding both orthologs with exceptionally high affinity, MYD1-72 overcomes this challenge, and thus the efficacy obtained in our mouse models is more representative of what could be achieved clinically.

One major challenge when interpreting the results of preclinical models is that their multivariable nature precludes an accurate assessment of efficacy in the absence of a known, internal control.
Therefore, to more appropriately determine its clinical potential, we placed MYD1-72 head to head with leading anti-AXL TKIs. We demonstrate that, in vitro, MYD1-72 Fc specifically inhibits AXL signaling without affecting MER or TYRO3 activities, whereas the TKIs were more promiscuous. Foretinib and BGB324 are both known as AXL inhibitors, though their ability to attenuate AXL signaling differs dramatically. At the same dosage, foretinib was indiscriminate, inhibiting all 3 TAM receptor family members, whereas BGB324 treatment resulted only in modest inhibition of AXL signaling. In all cases, downstream pAkt expression was suppressed, suggesting that BGB324 can elicit its therapeutic efficacy by influencing alternative signaling cascades. These different activity profiles are likely due to two critical factors: MYD1-72 Fc only binds to GAS6, leaving proteins and other ligands present in the serum free to bind to MER and TYRO3, and the polypharmacology of the anti-AXL TKIs results in broader kinase inhibition.

One critical detail of this study was the dosages that were chosen. Initial studies outlining the development of BGB324 achieved efficacy at $7 \mathrm{mg} / \mathrm{kg}$ twice daily, but other studies more consistently used dosages in excess of $100 \mathrm{mg} / \mathrm{kg}$ twice daily (26). This high dose is in line with what is often used when evaluating TKIs preclinically, most likely because a negative result at these elevated doses would be definitive. However, clinically, the total daily dose of approved TKIs in oncology is, with few exceptions, less than 12 $\mathrm{mg} / \mathrm{kg}$ (Supplemental Table 5). Though differences in how a drug behaves in mice and human subjects invariably exist, doses that exceed what is realistically achievable in the clinic by over an order of magnitude can skew preclinical results. As a result, we chose to dose both foretinib and BGB324 at $12.5 \mathrm{mg} / \mathrm{kg}$ twice daily. For MYD1-72 Fc, we dosed mice at $1 \mathrm{mg} / \mathrm{kg}$ daily. We have previously shown that this dosing regimen is equivalent to $10 \mathrm{mg} / \mathrm{kg}$ administered biweekly and thus chose daily dosing, as it reduced the total protein administered by nearly two-thirds.

When directly compared, the efficacy of MYD1-72 Fc was equivalent to that of the best TKI tested, foretinib. However, severe toxicity undermined foretinib's results, as half of the treatment group was removed from the study prior to the predetermined endpoint due to excessive morbidity. This highlights one of the confounding issues of using elevated doses of TKIs; the high homology of kinases results in off-target activity of even the most specific inhibitors. This promiscuity was observed in the clinic as well. In a recent phase II trial, foretinib failed to demonstrate efficacy despite the observation of treatment-related adverse events in $91 \%$ of patients (42). Furthermore, within our study, BGB324 was largely ineffective when dosed at $12.5 \mathrm{mg} / \mathrm{kg}$ twice a day, having only a marginal effect on metastatic disease. It should be

\section{Table 2. Treatment survival}

$\begin{array}{lc}\text { Treatment } & \text { Median survival (days) } \\ \text { Vehicle } & 17 \\ \text { MYD1-72 Fc } & 17 \\ \text { Cemcitabine } & 35 \\ \text { Combination } & 57\end{array}$




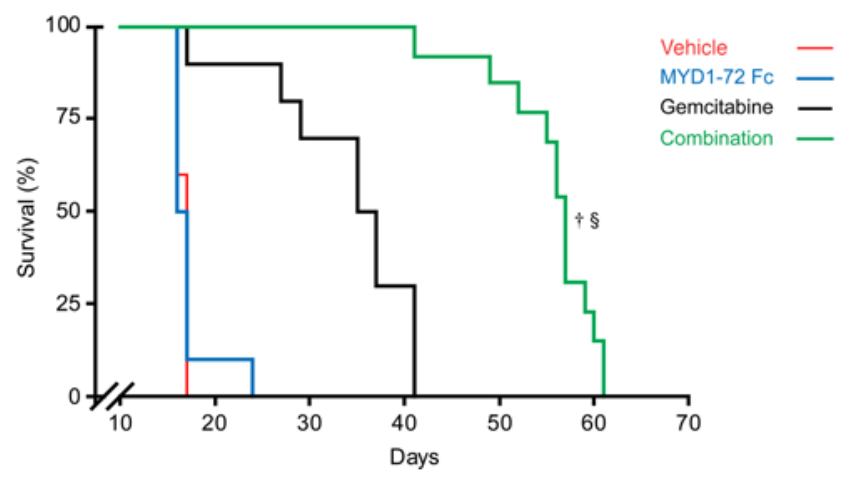

Figure 7. Inhibition of $A X L$ in pancreatic cancer prolongs survival when used in combination with gemcitabine. Kaplan-Meier of the LM-P orthotopic pancreatic cancer study. Animals were treated with either vehicle, MYD1-72 Fc, gemcitabine, or MYD1-72 Fc plus gemcitabine. ${ }^{\circledR} P<0.0001$ for combination vs. vehicle; ${ }^{\dagger} P<0.0001$ for combination vs. gemcitabine. A log-rank (Mantel-Cox) test was performed to compare mean survival among groups.

reiterated that this dose of BGB324 is on the low end of what has been reported to be effective. The studies on clinical dose escalation have not been published, and it is possible that higher doses of BGB324 would be effective, should they prove to be tolerable. Collectively, these data demonstrate that the specificity of our biologic allows marked antitumor activity to be achieved with little toxicity. These effects are equivalent to what can be attained by TKIs at their maximally tolerated dose, but carry few, if any, of the off-target risks of those compounds.

An unexpected result from the $4 \mathrm{~T} 1$ study was that treatment with MYD1-72 Fc substantially reduced primary tumor growth. Immunohistochemical analysis of primary tumor samples showed elevated levels of $\gamma \mathrm{H} 2 \mathrm{AX}$, leading us to investigate the link between AXL and the DNA-damage response. Using an RPPA, we uncovered a new relationship between AXL signaling and the DNA-damage response, wherein inhibition of AXL resulted in increased levels of DNA-damage markers such as CHK2, XRCC1, BAX, and BID. Furthermore, we demonstrated that the loss of AXL signaling upon treatment with MYD1$72 \mathrm{Fc}$ leads to phosphorylation of the classical DNA-damage response components ATM, ATR, and RPA32. By performing an EdU incorporation assay with $\mathrm{Hu}$ and MYD1-72 Fc, we are proposing a new role for AXL signaling in protecting cancer cells from disruptive replication and fork collapse during the $S$ phase. Based on these data, we concluded that the GAS6/ AXL signaling cascade provides cytoprotection for tumor cells and loss of AXL removes this protection, leading to replication stress and subsequent activation of the DNA-damage response. We showed that these effects were more pronounced when AXL was inhibited on cells cultured under serum-limited conditions, as cells grown in complete growth media showed a negligible increase in $\gamma \mathrm{H} 2 \mathrm{AX}$ expression upon treatment with MYD1-72 Fc. This observation is insightful, as it indicates that AXL's protective role is only activated under stress. This provides context to the fact that upregulation of AXL is generally associated with later stage metastatic disease, situations where tumor cells are under severe nutrient and oxygen deprivation.
DNA-damaging agents have long been used to exploit cancer's hyperproliferative state, and modulation of the damage response through the abrogation of AXL signaling was shown to increase the therapeutic effects of these drugs both in vitro and in vivo. In fact, this effect was so pronounced that the reduction of tumor burden in models of human ovarian cancer exceeded $99 \%$ and in some cases resulted in complete cures. A confounding aspect of our studies is that substantial differences exist between the in vivo models used, making direct comparisons less straightforward. However, that these effects were observed in multiple tumor models suggests that the relationship between AXL and the DNAdamage response is one that transcends cancer subtypes. These results offer the possibility of improving the therapeutic index of many standard-of-care chemotherapies, potentially providing a meaningful advancement in the care of many patients with refractory disease. Moving forward, additional studies exploring whether other DNA-damaging modalities, such as radiation, would benefit from concurrent AXL inhibition are justified. Furthermore, clinical studies combining candidate AXL inhibitors with cytotoxic agents would provide a clear advantage when evaluating efficacy.

Collectively, this study expands the role that AXL plays in tumor progression and the development of therapeutic resistance. By implicating the receptor as an important regulator of the DNA-damage response, there is the exciting possibility that anti-AXL therapies can be broadly used to chemosensitize in a myriad of malignancies. Our second-generation AXL decoy receptor is poised to capitalize upon this potential by providing a molecule capable of effectively antagonizing the GAS6/AXL system, with little to no toxicity. Clinical evaluation of MYD1-72 $\mathrm{Fc}$ is therefore justified, particularly when used as an adjuvant to standard-of-care chemotherapy.

\section{Methods}

Recombinant protein production. AXL Ig1 variants were produced in the methylotrophic yeast Pichia pastoris, as previously described (17). Briefly, Ig1 variants were cloned into the pPIC9K plasmid (no. V17520, Thermo Fisher Scientific) with flanking $\mathrm{N}$ - and C-terminal FLAG and $6 \mathrm{xHIS}$ tags, respectively, and yeast were transformed according to the manufacturer's protocol. Recombinant proteins were purified from conditioned culture supernatant by nickel affinity chromatography followed by removal of N-linked glycans using endoglycosidase H (EndoHf, P0703S, New England Biolabs). Final purification was performed using size-exclusion chromatography. Human GAS6 LG1-2 and both AXL constructs used for crystallography studies were expressed and purified as previously described. All AXL Fc fusion constructs were expressed transiently using the FreeStyle Max (Invitrogen) HEK293 system as previously described. Dimeric Fc fusions were isolated from conditioned culture supernatant using protein A affinity chromatography followed by size exclusion chromatography.

The Kinetic Exclusion Assay. Equilibrium binding and association rate constants for all GAS6-AXL interactions were measured on a Kinetic Exclusion Assay instrument (KinExA, Sapidyne Instruments Inc.) as previously described (17).

For equilibrium binding studies, 3 independent titrations were completed for each AXL Ig1 or AXL Fc fusion unless otherwise noted. Depending on the affinity of interaction, 3 of the following 4 titrations were performed: (a) $1 \mathrm{ml}$ reactions of $5 \mathrm{nM}$ GAS6, AXL serially dilut- 
A

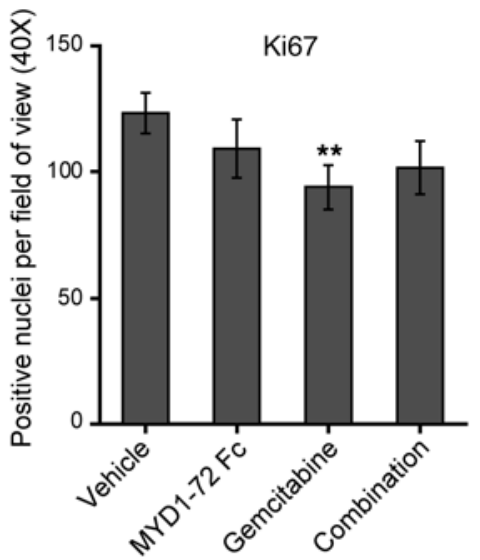

B

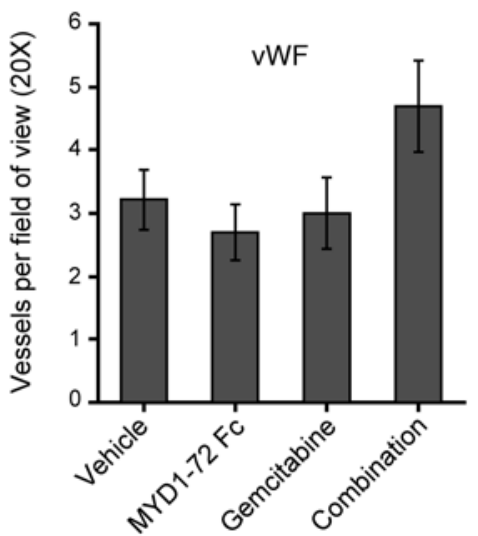

C

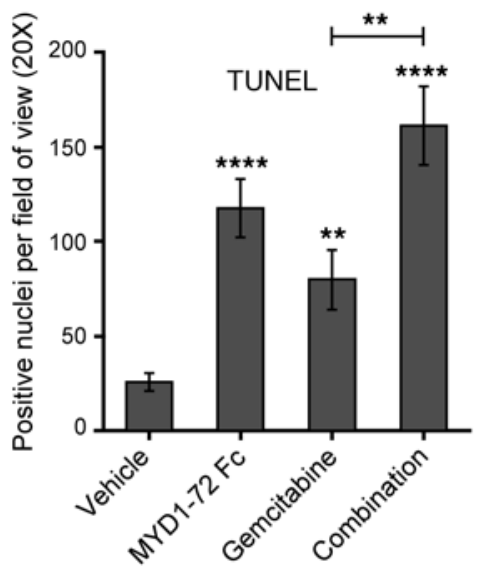

D

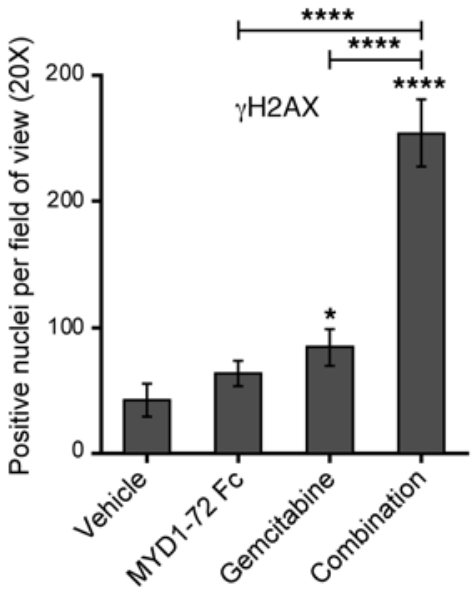

Vehicle

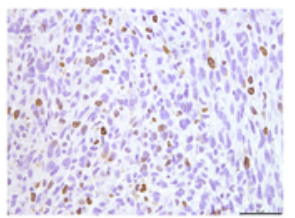

MYD1-72 Fc
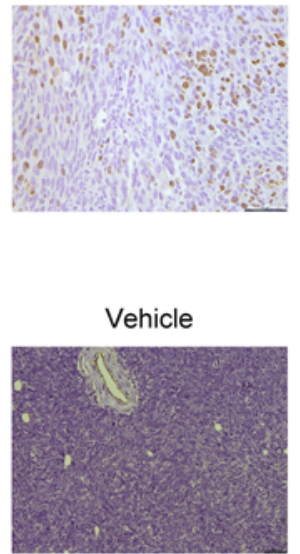

MYD1-72 Fc

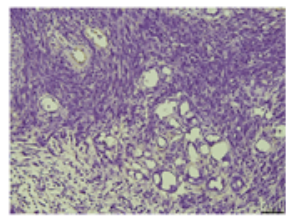

Vehicle

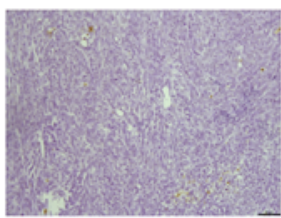

MYD1-72 Fc

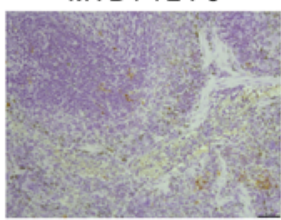

Vehicle

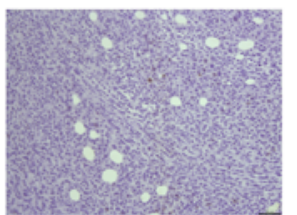

MYD1-72 Fc

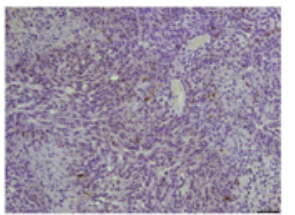

Gemcitabine

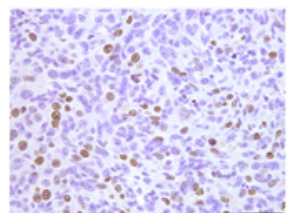

Combination

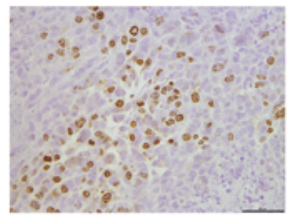

Gemcitabine

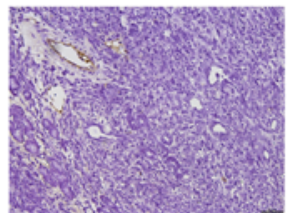

Combination

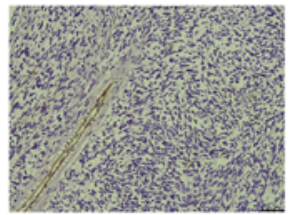

Gemcitabine

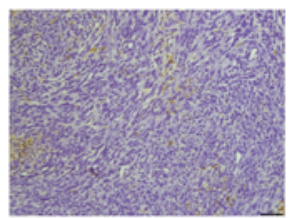

Combination

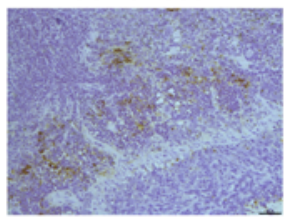

Gemcitabine

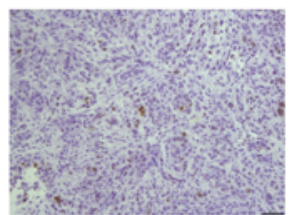

Combination

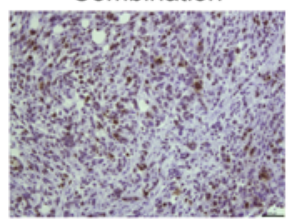

Figure 8. Inhibition of AXL in pancreatic cancer improves the efficacy of gemcitabine. Representative images and matched quantification of (A) Ki67, (B) vWF, (C) TUNEL, and (D) $\gamma \mathrm{H} 2 \mathrm{AX}$ staining of primary tumor tissue. Error bars represent mean \pm SD. $n=10-14 .{ }^{*} P<0.05 ;{ }^{* *} P<0.01$; ${ }^{* * * *} P<0.0001$. Scale bars: $50 \mu \mathrm{m}$. ANOVA with Tukey-Kramer test was used for comparing multiple treatments to each other.

ed 1:2 twelve times starting at $30 \mathrm{nM}, 3$ hours RT incubation; (b) $2 \mathrm{ml}$ reactions of $500 \mathrm{pM}$ GAS6, AXL serially diluted 1:2.5 twelve times starting at $10 \mathrm{nM}, 18$ hours RT incubation; (c) $12 \mathrm{ml}$ reactions of 50 pM GAS6, AXL serially diluted 1:3 twelve times starting at $9 \mathrm{nM}, 1$ day RT incubation; (d) $12 \mathrm{ml}$ reactions of $15 \mathrm{pM}$ GAS6, AXL serially diluted 1:3 twelve times starting at $1 \mathrm{nM}, 4$ day RT incubation. After the appropriate incubation time, reactions were flowed over MYD1 Fc-coated beads and captured free GAS6 was probed using an anti-6xHIS Dylight 649 Ab (no. 200-343-382, Rockland Immunochemicals Inc.). Each sample was measured twice, and data from the 3 independent equilibrium binding experiments were globally analyzed using n-curve analysis in the KinExA Pro 3.6.2 software (Sapidyne Instruments Inc.) to obtain the $K_{D}$ value.

To analyze the association rate of the interactions, the direct inject method was used. For these experiments, $1 \mu \mathrm{M}$ AXL was serially diluted 1:2.5, and equal volumes of each AXL sample and 500 nM GAS6 were briefly mixed and flowed over the capture beads. Free GAS6 was detected as described, and the data were fit using the KinExA Pro 3.6.2 software to obtain the association rate $\left(K_{o n}\right)$ of the interaction. The dissociation rate $\left(K_{o f f}\right)$ was calculated as the product of the $K_{D}$ and the $K_{o n}$.

Circular dichroism spectroscopy. The circular dichroism spectra of AXL Ig1 variants were measured on a Jasco J-815 circular dichroism spectropolarimeter. Recombinant proteins were diluted to $10 \mu \mathrm{M}$ in PBS, and ellipticity was measured in the far UV range from $190-260 \mathrm{~nm}$ at $20^{\circ} \mathrm{C}$ in a quartz cuvette with a 1-mm path length. Raw data were transformed to mean residue ellipticity using the following equation: $[\theta]_{m r w}=\left(m r w \times \theta_{o b s}\right) /(10 \times l \times c)$, where $m r w$ is the mean molecular weight per residue, $\theta_{o b s}$ is observed ellipticity in degrees, $l$ is the path length in centimeters, and $c$ is the concentration of protein in grams $/ \mathrm{ml}$. Three independent data sets were collected and averaged to obtain the spectrum for each protein, with each data set representing the average of triplicate scans. 
Crystallization and data collection of GAS6/AXL cocomplexes. Crystallization for the 2:2 GAS6/MYD1-72 Ig1-2 cocomplex was performed as previously described (22). Briefly, purified WT GAS6 and MYD1-72 Ig1-2 were mixed in a 1:1 molar ratio and allowed to complex at room temperature for 24 hours. The cocomplexes were purified using size exclusion chromatography to remove any unreacted components and were buffer exchanged into $25 \mathrm{mM}$ Na-HEPES, $150 \mathrm{mM}$ $\mathrm{NaCl}$, and $1 \mathrm{mM}$ calcium acetate to a final concentration of $10 \mathrm{mg} /$ $\mathrm{ml}$. Crystals for the GAS6/MYD1-72 cocomplex were grown at room temperature by the hanging-drop vapor-diffusion method with a 1:1 mixture $(1.2 \mu \mathrm{l}$ each) of the complex solution $(5.6 \mathrm{mg} / \mathrm{ml})$ and the well solution containing $0.15 \mathrm{M} \mathrm{Li}_{2} \mathrm{SO}_{4}, 0.1 \mathrm{M}$ Tris- $\mathrm{HCl}$ (pH 8.5), $5 \%$ glycerol, and $2 \mathrm{mM} \mathrm{Ni}_{2} \mathrm{SO}_{4}$. For cryocooling, the crystals were dipped in a solution containing 38 parts of $1 \mathrm{M} \mathrm{Li}_{2} \mathrm{SO}_{4}, 0.1 \mathrm{M}$ Tris- $\mathrm{HCl}$ ( $\mathrm{pH}$ 8.5), 0.1 $\mathrm{M} \mathrm{NaCl}$, and 12 parts of $100 \%$ glycerol. Diffraction data sets for the GAS6/MYD1-72 cocomplex (3.5Å) were collected at $100 \mathrm{~K}$ using the Stanford Synchrotron Radiation Lightsource (SSRL) beamline 12-2 at a wavelength of $0.98 \AA$. Data were indexed and integrated using the XDS package. The crystals belong to space group P3, 21 and contain 2 monomers per symmetric unit. The crystallographic data are summarized in Supplemental Table 3. Several attempts to improve the resolution by using various additives, crystal dehydration experiments, and changes in the cryocooling procedure were unsuccessful.

Nearly identical conditions were used to crystalize the 1:1 GAS6/ MYD1-72 Ig1 cocomplex. Briefly, purified WT GAS6 and MYD1-72 Ig1 were mixed in a 1:1 molar ratio and allowed to complex at room temperature for 24 hours. The cocomplexes were purified using size exclusion chromatography to remove any uncomplexed components and buffer exchanged into $25 \mathrm{mM}$ Na-HEPES, $150 \mathrm{mM} \mathrm{NaCl}$, and 1 $\mathrm{mM}$ calcium acetate to a final concentration of $10 \mathrm{mg} / \mathrm{ml}$. Crystals for the GAS6/MYD1-72 Ig1 cocomplex were grown at room temperature by the hanging-drop vapor-diffusion method with a 1:1 mixture $(1.2 \mu \mathrm{l}$ each) of the complex solution $(9.8 \mathrm{mg} / \mathrm{ml})$ and the well solution containing $0.7 \mathrm{M} \mathrm{Li}_{2} \mathrm{SO}_{4}$ and $0.1 \mathrm{M}$ Tris- $\mathrm{HCl}(\mathrm{pH}$ 8.4). For cryocooling, the crystals were dipped in a solution containing 38 parts of $1.1 \mathrm{M} \mathrm{Li}_{2} \mathrm{SO}_{4}$, $0.1 \mathrm{M}$ Tris- $\mathrm{HCl}$ (pH 8.4), $0.1 \mathrm{M} \mathrm{NaCl}$, and 12 parts of $100 \%$ glycerol. Before cryocooling, crystals were slightly dehydrated by placing the cover slip over $1 \mathrm{M} \mathrm{Li}_{2} \mathrm{SO}_{4}, 0.1 \mathrm{M}$ Tris- $\mathrm{HCl}(\mathrm{pH}$ 8.4) well solution for 8 hours. The diffraction data set for the GAS6/MYD1-72 Ig1 cocomplex $(2.3 \AA)$ was collected at $100 \mathrm{~K}$ using the SSRL beamline $12-2$ at a wavelength of $0.98 \AA$. Data were indexed and integrated using the XDS package. The crystals belong to space group $\mathrm{P} 2{ }_{1}{ }_{2}{ }_{1}{ }_{1}$ and contain 2 monomers per symmetric unit. The crystallographic data are summarized in Supplemental Table 3. Crystals were also grown from 3 other crystallization conditions, but they showed weaker diffraction.

Structure determination and refinement. For the GAS6/MYD1-72 Ig1-2 cocomplex, initial phases were obtained by molecular replacement by using the program MOLREP and the coordinates of the WT GAS6/AXL crystal structure (PDB ID:2C5D) as the search model. Several cycles of manual model building using COOT (43) and refinement using REFMAC (44) resulted in final $R_{\text {working }}$ and $R_{\text {free }}$ values of $20.3 \%$ and $24.5 \%$, respectively. The Ramachandran statistics are as follows: 93.28 (favored) and 1.15 (outlier). The refinement statistics are provided in Supplemental Table 3. The structure of the GAS6/MYD1-72 Ig1 cocomplex was similarly solved by molecular replacement, again using the coordinates of the WT structure as the search model. No electron density was observed for the loop of GAS6 from residues 542 to 550 in both chain A and chain B for the GAS6/MYD1-72 Ig1 cocomplex; the WT GAS6/AXL Ig1-2 crystal (PDB ID: 2C5D) structure also did not show electron density for this region. Several cycles of manual model building using COOT and refinement using REFMAC resulted in final $R_{\text {working }}$ and $R_{\text {free }}$ values of $19.8 \%$ and $24.7 \%$, respectively. The Ramachandran statistics are as follows: $96.6 \%$ (favored) and $0.64 \%$ (outlier). The refinement statistics are provided in Supplemental Table 3. All crystal structure figures were created using PyMOL (45).

Analysis of intermolecular contacts. The intermolecular contacts between GAS6 and MYD1 A72V in the cocomplex were examined as described. Briefly, the binding interface was analyzed using the PDBePISA (46) server v1.47 (http://www.ebi.ac.uk/pdbe/prot_int/ pistart.html) utilizing a cutoff of $3.5 \AA$ and 4.0 A for hydrogen bonds and electrostatic interactions, respectively. Analysis was performed in a manner identical to that used with the prior GAS6/MYD1 structure to enable direct comparison.

Analysis of van der Waals contacts. The van der Waals contacts between the side chain of the residue at position 72 and surrounding residues were determined using the WHAT IF server. A contact is defined as 2 atoms for which the distance between the van der Waals surfaces is less than $1.0 \AA$. The WHAT IF algorithm uses the following

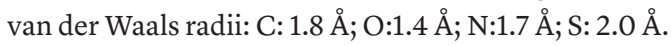

In vivo tumor models. For all in vivo studies, 6-week-old, female nude (nu/nu) mice (Jackson Laboratory) were used. In all studies, animals displaying signs of morbidity were removed from the study immediately.

To establish orthotopic mammary tumors, $5 \times 10^{4} 4 \mathrm{~T} 1$ luciferase cells (47) (a gift from Marta Vilalta, Stanford University) were injected in a volume of $50 \mu \mathrm{l}$ of DMEM into the mammary fat pad. Tumor engraftment was confirmed 4 days after tumor inoculation by bioluminescence imaging using an IVIS 200 (PerkinElmer). Treatment was initiated 4 days after inoculation for both studies. In the initial study comparing MYD1 Fc and MYD1-72 Fc, proteins were administered i.v. twice a week via tail vein injection in a volume of $200 \mu \mathrm{l}$ at $0.5 \mathrm{mg} /$ $\mathrm{kg}$. In the second study comparing MYD1-72 Fc and the small molecule TKIs, proteins were administered daily via i.p. injections at $1 \mathrm{mg} /$ kg. BGB324 (Selleckchem LLC) and foretinib (MedChem) were given twice daily via oral gavage at $12.5 \mathrm{mg} / \mathrm{kg}$.

Small molecules were administered twice daily via oral gavage. On day 24, mice were sacrificed 10 minutes after receiving a single i.p. injection of D-luciferin. Ex vivo bioluminescent imaging of the lungs and spleen was performed using an IVIS 200, and images were analyzed using Living Image software v4.3.1 to quantify lung metastases.

In both the skov3.ip (a gift from Gordon Mills at MD Anderson Cancer Center, Houston, Texas, USA) and OVCAR8 ovarian cancer models (NCI-Frederick Division of Cancer Treatment and Diagnosis [DCTD] tumor cell line repository), diffuse metastatic disease was established by injecting $1 \times 10^{6}$ cells i.p. Tumors were allowed to establish for 1 week prior to treatment, and on day 5 , a subset of animals were sacrificed to confirm the presence of visible macroscopic disease. In both studies, MYD1-72 Fc was administered at $1 \mathrm{mg} / \mathrm{kg}$ i.p. daily, doxorubicin (APP Pharmaceuticals) at $2 \mathrm{mg} / \mathrm{kg}$ was administered i.p. twice a week, or both were administered as described for a total of 3 weeks. Animals were sacrificed on day 28 , and metastatic burden was assessed by counting the number of visible metastatic lesions in the peritoneal cavity as well as excising and weighing all tumor tissue.

The orthotropic LM-P pancreatic adenocarcinoma model (a gift from Edgar Engleman at Stanford University ) was established as previ- 
ously described (38). Briefly, $1 \times 10^{6}$ cells were injected s.c. into the flanks of nude mice and grown for 2 to 3 weeks until they reached $500 \mathrm{~mm}^{3}$. To establish orthotropic tumors, mice harboring the s.c. tumors were sacrificed and tumors were isolated and cut into small 3- to 4-mm fragments. Laparotomies were performed, and a tumor fragment was secured to the tail of the pancreas using sutures. After implantation, the pancreas was returned to the peritoneal cavity and the incision was closed. Mice received daily injections of antibiotics on the day of implantation and on each of the 3 days after the operation for pain management. Treatment was initiated 4 days after surgery. MYD1-72 Fc was administered at 1 $\mathrm{mg} / \mathrm{kg}$ i.p. daily, gemcitabine (Sun Pharmaceutical Industries Inc.) at 2 $\mathrm{mg} / \mathrm{kg}$ was administered i.p. twice a week, or both were administered as described. Treatment was continued until a mouse displayed signs of morbidity, at which time it was removed from the study.

In vivo GAS6 serum ELISA. For the time course studies, mice were administered a single dose of MYD1 Fc or MYD1-72 Fc at $0.5 \mathrm{mg} / \mathrm{kg}$ via tail vein injection. All doses were formulated in a $200 \mu$ volume. Two mice were analyzed per condition, and untreated mice were used to determine baseline GAS6 levels. At 2, 12, 24, and 36 hours after administration, retroorbital bleeds were performed to obtain blood samples from which serum was isolated and free GAS6 was measured as previously described. The amount of free GAS6 in each sample was determined using a sandwich ELISA. In this assay, MYD1 Fc was used as a capture reagent in order to ensure the detection of free, unbound GAS6 and not GAS6/AXL Fc complexes. Detection of GAS6 was carried out using a biotinylated polyclonal antimouse GAS6 Ab (BAF986, R\&D Systems) and streptavidin HRP (no. 4800-30-06, Trevigen Inc.).

$R P P A$. The RPPA was performed by MD Anderson as described.

Immunoblotting. Cell lysates were subjected to sodium dodecyl sulfate polyacrylamide gel electrophoresis, followed by transfer to nitrocellulose membrane. The membranes were then probed with primary Abs against total AXL (AF154, R\&D Systems), pAXL (T702; no. 5724, CST), total Akt (no. 4691 CST), pAkt (no. 4060, CST), anti-MER (no. ab52968, Abcam), pMER (no. ab14921 Abcam), anti-TYRO3 (no. 5585, CST), pTyro3 (no. orb186274, Biorbyt LLC), BCL-2 Family Antibody Sampler Kit (no. 9934, CST), anti-CHK2 (no. sc-5278, Santa Cruz Biotechnology Inc.), anti-XRCC1 (no. 2735, CST), anti- $\gamma$ H2AX (no. 05636, Merck Millipore), anti-pATM (no. ab81292, Abcam), anti-ATM (no. ab78, Abcam), anti-pATR (no. 2853, CST), anti-ATR (no. 2790, CST), anti-phospho CHK1 (no. 12302, CST), anti-pHK2 (no. 2197, CST) and anti-RPA32 (no. 2208, CST) at $4^{\circ} \mathrm{C}$ overnight. The blots were then washed and probed with HRP-conjugated anti-goat (no. sc-2020, Santa Cruz Biotechnology Inc.), or HRP-conjugated anti-rabbit (no. A16110, Thermo Fisher Scientific) as appropriate. The blots were developed with Bio-Rad Western C Developing Reagent (no. 170-5060 Bio-Rad) and visualized with Chemidoc digital imager (no. 1708280, Bio-Rad).

Immunofluorescence analysis. 10,000 cells were plated in each well of glass chamber slide and allowed to attach overnight. Cells were serum starved and treated with $0.1 \mu \mathrm{g}$ or $10 \mu \mathrm{g}$ of MYD1-72 Fc with or without $(2.5 \mathrm{mg})$ doxorubicin. Cells were then washed with PBS and fixed with $4 \%$ paraformaldehyde, lysed for 10 minutes, and blocked with a $2 \%$ BSA and $0.1 \%$ PBS-Triton-X solution for 1 hour. After washes in PBS-Triton-X 100, cells were incubated with mouse anti- $\gamma \mathrm{H} 2 \mathrm{AX}$ (no. 05-636, Merck Millipore), rabbit anti-RAD51 (no. PC130-100UL Merck Millipore), or rabbit anti-53BP1 (no. ab36823, Abcam) overnight in a humidified chamber at $4^{\circ} \mathrm{C}$. Cells were washed with PBS and incubated in secondary Ab anti-rabbit FITC (no. 65-6111, Thermo Fisher
Scientific) or anti-mouse FITC (no. 62-6511, Thermo Fisher Scientific) for 1 hour at $37^{\circ} \mathrm{C}$ and counterstained with Prolong Mounting Medium with DAPI. Positive foci intensities were calculated using Imagine Software Metamorph. EdU (5-ethynyl-2'-deoxyuridine) labeling and costaining were carried out according to the manufacturer's instructions (no. C10637, Thermo Fisher Scientific). $\gamma \mathrm{H} 2 \mathrm{AX}$ staining was performed as described above. Cells were visualized using a Leica DM6000 B microscope with Leica Application Suite X software. EdU was used at a concentration of $10 \mu \mathrm{M}$. EdU labeling was carried out for 1 hour in all cases; for conditions including drug treatments, EdU was added during the last hour of treatment. Experiments were carried out in triplicate. Quantification of staining: positive $\gamma \mathrm{H} 2 \mathrm{AX}$ staining refers to cells with over 8 foci and pan-nuclear staining. For Hu-treated groups, $\gamma \mathrm{H} 2 \mathrm{AX}$-positive cells refers to cells where a pannuclear staining pattern (typically associated with replication stress) was observed.

Statistics. ANOVA with Tukey-Kramer test was used for comparing multiple treatment groups with each other. $P<0.05$ was considered significant. Repeated measure ANOVA was used for comparing multiple treatment groups measured over time. Statistical analysis of survival curves was conducted in the pancreatic cancer survival study. A log-rank (Mantel-Cox) test was performed to compare mean survival among groups; $P \leq 0.05$ was considered statistically significant.

Study approval. All procedures involving animals and their care and use were approved by the Institutional Animal Care and Usage Committee of Stanford University.

\section{Author contributions}

All authors contributed to the design of the research. MSK performed the protein engineering and characterization; MSK, SK, and IIM did the crystallography; MSK, YRM, SEN, and AD conducted the in vivo experiments; all authors analyzed the data; MSK and AJG prepared the manuscript with input from all coauthors.

\section{Acknowledgments}

The authors would like to thank the Stanford FACS Core Facility for assistance with the flow cytometric sorting. Rie von Eyben helped with statistical analysis. All ex vivo imaging was conducted in the Stanford Small Animal Imaging Facility. Portions of this research were performed at the Stanford Synchrotron Radiation Laboratory, a national user facility operated by Stanford University on behalf of the U.S. Department of Energy Office of Basic Energy Sciences. This work was supported by the Silicon Valley Foundation, the Kimmelman Fund, the Skippy Frank Foundation, the Wallace H. Coulter Translational Research Grant Program (to JRC and AJG), the Stanford Chemistry, Engineering \& Medicine for Human Health (ChEM-H) Institute (to JRC and IIM), Stanford Bio-X and ARCS graduate fellowships (to MSK), NCI grant 88480 (to AJG and YRM), NIH training grant T32 GM008412-15S1, and a Siebel graduate fellowship (to DSJ). MMO is a Cancer Research Institute Irvington Fellow supported by the Cancer Research Institute.

Address correspondence to: Amato J. Giaccia, CCSR-South, Room 1255, 269 Campus Drive, Stanford, California 94305-5152, USA. Phone: 650.723.7311; E-mail: giaccia@stanford.edu. Or to: Jennifer R. Cochran, Shriram Center for Bioengineering and Chemical Engineering, 443 Via Ortega, Room 356, Stanford, California 943054125, USA. Phone: 650.724.5034; E-mail: cochran@stanford.edu. 
1. Scott AM, Wolchok JD, Old LJ. Antibody therapy of cancer. Nat Rev Cancer. 2012;12(4):278-287.

2. Fabian MA, et al. A small molecule-kinase interaction map for clinical kinase inhibitors. Nat Biotechnol. 2005;23(3):329-336.

3. Wan L, Pantel K, Kang Y. Tumor metastasis: moving new biological insights into the clinic. Nat Med. 2013;19(11):1450-1464.

4. Lemke G, Rothlin CV. Immunobiology of the TAM receptors. Nat Rev Immunol. 2008;8(5):327-336.

5. O'Bryan JP, et al. axl, a transforming gene isolated from primary human myeloid leukemia cells, encodes a novel receptor tyrosine kinase. $\mathrm{Mol}$ Cell Biol.1991;11(10):5016-5031.

6. Linger RM, Keating AK, Earp HS, Graham DK. TAM receptor tyrosine kinases: biologic functions, signaling, and potential therapeutic targeting in human cancer. Adv Cancer Res. 2008;100:35-83.

7. Li Y, et al. Axl as a potential therapeutic target in cancer: role of Axl in tumor growth, metastasis and angiogenesis. Oncogene. 2009;28(39):3442-3455.

8. Rankin EB, et al. AXL is an essential factor and therapeutic target for metastatic ovarian cancer. Cancer Res. 2010;70(19):7570-7579.

9. Vajkoczy P, et al. Dominant-negative inhibition of the Axl receptor tyrosine kinase suppresses brain tumor cell growth and invasion and prolongs survival. Proc Natl Acad Sci U S A. 2006;103(15):5799-5804.

10. Chen PX, Li QY, Yang Z. Axl and prostasin are biomarkers for prognosis of ovarian adenocarcinoma. Ann Diagn Pathol. 2013;17(5):425-429.

11. Gjerdrum C, et al. Axl is an essential epithelialto-mesenchymal transition-induced regulator of breast cancer metastasis and patient survival. Proc Natl Acad Sci U S A. 2010;107(3):1124-1129.

12. Zhang Z, et al. Activation of the AXL kinase causes resistance to EGFR-targeted therapy in lung cancer. Nat Genet. 2012;44(8):852-860.

13. Hong J, Peng D, Chen Z, Sehdev V, Belkhiri A. ABL regulation by AXL promotes cisplatin resistance in esophageal cancer. Cancer Res. 2013;73(1):331-340.

14. Lu Q, et al. Tyro-3 family receptors are essential regulators of mammalian spermatogenesis. Nature. 1999;398(6729):723-728.

15. Nagata K, et al. Identification of the product of growth arrest-specific gene 6 as a common ligand for Axl, Sky, and Mer receptor tyrosine kinases. J Biol Chem. 1996;271(47):30022-30027.

16. Varnum BC, et al. Axl receptor tyrosine kinase stimulated by the vitamin K-dependent protein encoded by growth-arrest-specific gene 6 . Nature. 1995;373(6515):623-626.

17. Kariolis MS, et al. An engineered Axl 'decoy receptor' effectively silences the Gas6-Axl signaling axis. Nat Chem Biol. 2014;10(11):977-983.

18. Economides AN, et al. Cytokine traps: multi-component, high-affinity blockers of cyto- kine action. Nat Med.2003;9(1):47-52.

19. Kariolis MS, Kapur S, Cochran JR. Beyond antibodies: using biological principles to guide the development of next-generation protein therapeutics. Curr Opin Biotechnol. 2013;24(6):1072-1077.

20. Peppel K, Crawford D, Beutler B. A tumor necrosis factor (TNF) receptor-IgG heavy chain chimeric protein as a bivalent antagonist of TNF activity. JExp Med.1991;174(6):1483-1489.

21. Holash J, et al. VEGF-Trap: a VEGF blocker with potent antitumor effects. Proc Natl Acad Sci U S A. 2002;99(17):11393-11398.

22. Sasaki T, et al. Structural basis for Gas6-Axl signalling. EMBO J. 2006;25(1):80-87.

23. Chao G, Lau WL, Hackel BJ, Sazinsky SL, Lippow SM, Wittrup KD. Isolating and engineering human antibodies using yeast surface display. Nat Protoc. 2006;1(2):755-768.

24. Park IK, Mishra A, Chandler J, Whitman SP, Marcucci G, Caligiuri MA. Inhibition of the receptor tyrosine kinase Axl impedes activation of the FLT3 internal tandem duplication in human acute myeloid leukemia: implications for Axl as a potential therapeutic target. Blood. 2013;121(11):2064-2073.

25. Thiede $\mathrm{C}$, et al. Analysis of FLT3-activating mutations in 979 patients with acute myelogenous leukemia: association with FAB subtypes and identification of subgroups with poor prognosis. Blood. 2002;99(12):4326-4335.

26. Holland SJ, et al. R428, a selective small molecule inhibitor of Axl kinase, blocks tumor spread and prolongs survival in models of metastatic breast cancer. Cancer Res. 2010;70(4):1544-1554.

27. Kataoka Y, et al. Foretinib (GSK1363089), a multi-kinase inhibitor of MET and VEGFRs, inhibits growth of gastric cancer cell lines by blocking inter-receptor tyrosine kinase networks. Invest New Drugs. 2012;30(4):1352-1360.

28. Zillhardt M, et al. Foretinib (GSK1363089), an orally available multikinase inhibitor of c-Met and VEGFR-2, blocks proliferation, induces anoikis, and impairs ovarian cancer metastasis. Clin Cancer Res. 2011;17(12):4042-4051.

29. Ruan GX, Kazlauskas A. Axl is essential for VEGF-A-dependent activation of PI3K/Akt. EMBO J. 2012;31(7):1692-1703.

30. D'Arcangelo D, Ambrosino V, Giannuzzo M, Gaetano C, Capogrossi MC. Axl receptor activation mediates laminar shear stress anti-apoptotic effects in human endothelial cells. Cardiovasc Res. 2006;71(4):754-763.

31. Rankin EB, et al. Direct regulation of GAS6/ AXL signaling by HIF promotes renal metastasis through SRC and MET. Proc Natl Acad Sci U S A. 2014;111(37):13373-13378.

32. Elkabets $\mathrm{M}$, et al. AXL mediates resistance to PI3K $\alpha$ inhibition by activating the EGFR/ $\mathrm{PKC} / \mathrm{mTOR}$ axis in head and neck and esophageal squamous cell carcinomas. Cancer Cell.
2015;27(4):533-546.

33. Bowtell DD, et al. Rethinking ovarian cancer II: reducing mortality from high-grade serous ovarian cancer. Nat Rev Cancer. 2015;15(11):668-679.

34. Burstyn-Cohen T, Lew ED, Través PG, Burrola PG, Hash JC, Lemke G. Genetic dissection of TAM receptor-ligand interaction in retinal pigment epithelial cell phagocytosis. Neuron. 2012;76(6):1123-1132.

35. Bosurgi L, et al. Paradoxical role of the protooncogene Axl and Mer receptor tyrosine kinases in colon cancer. Proc Natl Acad Sci U S A. 2013;110(32):13091-13096.

36. Cancer Facts and Figures 2014. American Cancer Society. http://www.cancer.org/acs/groups/ content/@research/documents/webcontent/ acspc-042151.pdf. Accessed October 19, 2016.

37. Li D, Xie K, Wolff R, Abbruzzese JL. Pancreatic cancer. Lancet. 2004;363(9414):1049-1057.

38. Tseng WW, et al. Development of an orthotopic model of invasive pancreatic cancer in an immunocompetent murine host. Clin Cancer Res. 2010;16(14):3684-3695.

39. Takeda Announces Phase 3 MONET-A Study Evaluating Motesanib (AMG 706) in Patients with Advanced Non-Squamous Non-Small Cell Lung Cancer Does Not Meet Primary Endpoint [press release]. Cambridge, MA; Osaka, Japan; Takeda; February 17, 2015. https://www. takeda.com/news/2015/20150217_6909.html. Accessed October 19, 2016.

40. Wu X, Liu X, Koul S, Lee CY, Zhang Z, Halmos B. AXL kinase as a novel target for cancer therapy. Oncotarget. 2014;5(20):9546-9563.

41. Ye X, et al. An anti-Axl monoclonal antibody attenuates xenograft tumor growth and enhances the effect of multiple anticancer therapies. Oncogene. 2010;29(38):5254-5264.

42. Shah MA, et al. Phase II study evaluating 2 dosing schedules of oral foretinib (GSK1363089), cMET/ VEGFR2 inhibitor, in patients with metastatic gastric cancer. PLoS One. 2013;8(3):e54014.

43. Emsley P, Cowtan K. Coot: model-building tools for molecular graphics. Acta Crystallogr D Biol Crystallogr. 2004;60(Pt 12 Pt 1):2126-2132.

44. Murshudov GN, Vagin AA, Dodson EJ. Refinement of macromolecular structures by the maximum-likelihood method. Acta Crystallogr D Biol Crystallogr. 1997;53(Pt 3):240-255.

45. Schrodinger, LLC. The PyMOL Molecular Graphics System, Version 1.3r1. PyMol. https://www. pymol.org/. Accessed October 19. 2016.

46. Krissinel E, Henrick K. Inference of macromolecular assemblies from crystalline state. J Mol Biol. 2007;372(3):774-797.

47. Aslakson CJ, Miller FR. Selective events in the metastatic process defined by analysis of the sequential dissemination of subpopulations of a mouse mammary tumor. Cancer Res. 1992;52(6):1399-1405. 